\title{
The role of non-uniqueness in the development of vortex breakdown in tubes
}

\author{
By PHILIP S. BERAN ${ }^{1}$ AND FRED E. C. CULICK \\ ${ }^{1}$ Department of Aeronautics and Astronautics, Air Force Institute of Technology, \\ Wright-Patterson AFB, OH 45433, USA \\ ${ }^{2}$ Jet Propulsion Center, Department of Aeronautics, California Institute of Technology, \\ Pasadena, CA 91125. USA
}

(Received 7 December 1990 and in revised form 10 January 1992)

Numerical solutions of viscous, swirling flows through circular pipes of constant radius and circular pipes with throats have been obtained. Solutions were computed for several values of vortex circulation, Reynolds number and throat/inlet area ratio, under the assumptions of steady flow, rotational symmetry and frictionless flow at the pipe wall. When the Reynolds number is sufficiently large, vortex breakdown occurs abruptly with increased circulation as a result of the existence of non-unique solutions. Solution paths for Reynolds numbers exceeding approximately 1000 are characterized by an ensemble of three inviscid flow types: columnar (for pipes of constant radius), soliton and wavetrain. Flows that are quasi-cylindrical and which do not exhibit vortex breakdown exist below a critical circulation, dependent on the Reynolds number and the throat/inlet area ratio. Wavetrain solutions are observed over a small range of circulation below the critical eirculation, while above the critical value, wave solutions with large regions of reversed flow are found that are primarily solitary in nature. The quasi-cylindrical $(\mathrm{QC})$ equations first fail near the critical value, in support of Hall's theory of vortex breakdown (1967). However, the QC equations are not found to be effective in predicting the spatial position of the breakdown structure.

\section{Introduction}

Vortex breakdown is a feature of rotational flows involving a concentrated core of vorticity embedded in a largely irrotational flow that is moving in a direction approximately parallel to the vortex. Examples of such flows are swirling flows through pipes and the vortical flows produced by delta wings at large angle-ofattack. 'Vortex breakdown' is the term used to identify the development of a stagnation point on a vortex core, followed by a limited region of reversed flow, with an associated dramatic increase of core size.

The study of vortex breakdown is important to the disciplines of both aerodynamies and combustion physics. The breakdown of the leading-edge vortices over a delta wing can have a significant effect on an aircraft's dynamics through large changes in the lift, drag and moment coefficients. It is thus desirable to be able to predict when breakdown will occur and at what position along the vortex it will develop. Faler \& Leibovich (1978) have also noted that it is important to understand the structure of the flow that develops as a consequence of vortex breakdown in some applications involving combustion chambers. In these applications, flows which lead 
to vortex breakdown, are purposefully generated so that the resulting bubble of recirculating fluid can be used as a 'fluid-dynamic flameholder'.

There have been several review papers on vortex breakdown, including the reports by Hall (1972), Leibovich (1978), Leibovich (1984) and Hall (1985).

The observation of a near-axisymmetric form of vortex breakdown ('bubble breakdown') by Lambourne \& Bryer (1961) has spawned a long series of experiments carried out by several investigators, involving the visualization of vortex breakdown in circular tubes. Vortices were generated by passing water through a set of swirl vanes near the entrance to a circular test section and visualized by introducing dye into the centre of the vortex, upstream of the test section. With this type of apparatus, Faler \& Leibovich (1977) observed and classified five types of vortex breakdown. The bubble form of breakdown, Faler \& Leibovich's type 0 , is 'characterized by a stagnation point on the swirl axis, followed by an abrupt expansion of the centerline dye filament to form the envelope of a bubble of recirculating fluid. The envelope has a high degree of axial symmetry over most of its length, but the rear is not closed and is asymmetric'. The last observation is common to all experimental studies of breakdown - some degree of asymmetry in the breakdown structure is always present.

In an effort to reproduce experimental results, investigations have been carried out to simulate bubble breakdown numerically with the assumption that the physical mechanisms responsible for this phenomenon are axisymmetric in nature. The first of these studies was carried out by Hall (1967), using the 'quasi-cylindrical' equations, a parabolized, approximate form of the Navier-Stokes equations. Hall's theory of vortex breakdown consists of the postulate that the position of vortex breakdown is given approximately by the point at which integration of the quasicylindrical equation fails. This situation is analogous to separation of a plane flow, where the approximate position of the separation point may be predicted by failure of the boundary-layer equations.

Kopecky \& Torrance (1973) were the first to model vortex breakdown using the Navier-Stokes equations. Since 1973, results of numerical simulation, using the Navier-Stokes equations, have been reported by Grabowski (1974); Krause, Shi \& Hartwich (1983); Hafez, Kuruvila \& Salas (1986); Beran (1987); Hafez et al. (1987); Brown \& Lopez (1988); Lopez (1988); Menne (1988); Beran (1989); Salas \& Kuruvila (1989). Each of these studies assumed the flow to be rotationally symmetric, thereby eliminating the possibility of studying the non-axisymmetric features of vortex breakdown, As emphasized by Faler \& Leibovich (1977), the term axisymmetric breakdown is a 'misnomer' since no breakdown form is truly axisymmetric. However, numerical simulations of internal, axisymmetric swirling flows have been quite successful in reproducing the breakdown structure. Computational results by Lugt \& Abboud (1987), Brown \& Lopez (1988) and Lopez (1988) were directly compared with flow visualizations recorded by Escudier (1984) and found to be very accurate simulations of the flow fields. Since the mechanism for breakdown in computed, axisymmetric flows is not well understood, a better understanding would most likely benefit any investigation of the three-dimensional phenomenon.

One important characteristic of most of the investigations which involve numerical simulation is that the computed solutions are unique. Three important exceptions are the studies of Táasan (1986), Hafez et al. (1986), and Leibovich \& Kribus (1990), where the breakdown of inviscid vortices in straight pipes was modelled. Solutions away from a trivial, columnar flow state were computed that exhibited reversed flow. Táasan and Hafez et al. computed a branch of non-trivial, solitary wave 


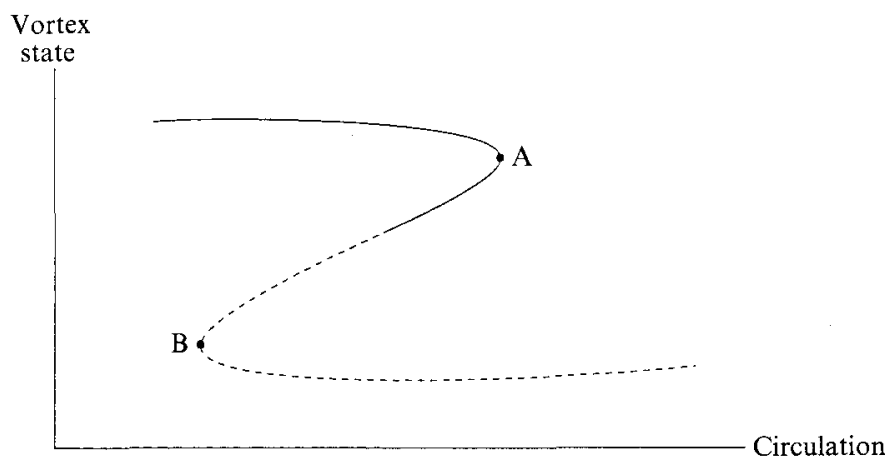

FIGURE 1. A representative solution diagram that relates vortex state to vortex circulation. A, primary limit point; B, secondary limit point; - - non-reversed flow, ---, reversed flow.

solutions that intersected the branch of trivial solutions at a simple bifurcation point. Leibovich \& Kribus confirmed the existence of this branch and also computed branches of wavetrain and non-unique, columnar solutions.

Hafez et al. (1986) computed solutions of the Navier-Stokes equations and found a rapid change in flow state over a small range of vortex strengths with $R e=200$. They could not determine, however, if the change was due to the presence of a hysteresis loop, implying non-uniqueness of flow states over a range of vortex strengths, or whether the change was simply due to the rapid growth of the breakdown structure in the absence of non-uniqueness. Also, Beran (1989) observed non-unique solutions of the Navier-Stokes equations for swirling flows in pipes, but found that the validity of many of these solutions was questionable owing to the presence of short wavelength errors. In neither of these studies was the potential impact of solution non-uniqueness in the Euler equations on the behaviour of viscous vortices described.

In the present study, non-uniqueness is observed when the Reynolds number is sufficiently large, and is manifested as the abrupt formation of reversed flow as the vortex circulation is increased through a critical value. In a diagram that shows a path of solution points as a function of circulation, non-uniqueness comes about through the formation of two limit points on the path, between which the vortex has three possible states. A sketch of this situation is provided in figure 1 . It is not the main purpose of this study to calculate breakdown structure, for such results have long been available. Instead, our chief contribution lies in the establishment of a connection between the appearance of reversed flow and the passage across a limit point as vortex circulation is increased. Although the process responsible for the formation of limit points has not been fully examined, the connection between such points and reversed flow may serve as a new departure point for the description of vortex breakdown.

Our examination of a one-parameter family of pipes with non-constant radius simplified the interpretation of computed results and provided a class of geometries more closely resembling that found in experiment (i.e. convergent, annular intake sections followed by slowly divergent test sections). Flow constriction downstream of the pipe inlet serves to desensitize the response of computed breakdown bubbles to changes in the boundary conditions applied at the inlet by reducing the magnitude of flow gradients in the vicinity of this boundary. In contrast, severe flow gradients near the inlet have been a difficulty in previous numerical investigations (e.g. 


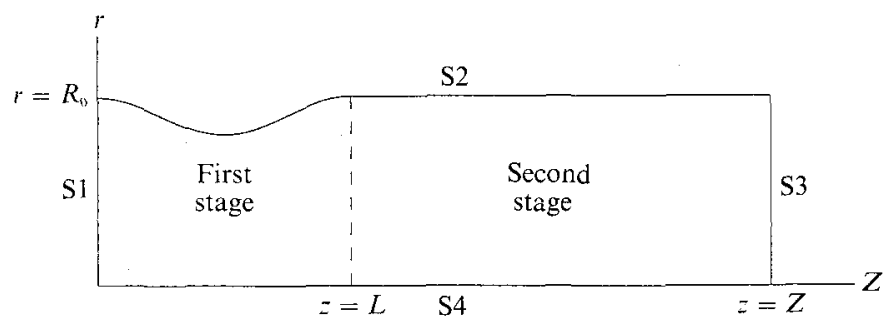

Figure 2. Schematic of pipe geometry.

Kopecky \& Torrance 1973; Grabowski 1974; Brown \& Lopez 1988) as a result of the general proximity of computed bubbles to the inlet. Furthermore, Beran (1989) reported that the structure and behaviour of the breakdown bubbles is sensitive to the choice of inflow conditions on azimuthal vorticity. Our approach has been to reduce flow gradients at the inlet by constraining the breakdown bubble to exist well downstream of the boundary, thereby reducing the sensitivity of the bubble to inflow conditions. The convergent section between the inlet and the pipe throat, as shown schematically in figure $\mathbf{2}$, experiences a favourable pressure gradient, and thus discourages vortex breakdown, while an adverse pressure gradient is promoted downstream of the throat where vortex breakdown is now encouraged. Our calculations revealed that only a slight deviation away from a straight pipe towards flow construction was necessary to constrain the breakdown bubble to lie downstream of the throat.

\section{Mathematical formulation}

\subsection{Assumptions}

The mathematical formulation of the problem of vortex breakdown in a pipe pursued in this study is based on several assumptions about the nature of the flow. The main assumptions are:

(i) incompressible flow,

(ii) rotationally symmetric ('axisymmetric') flow,

(iii) steady flow.

The assumption of incompressibility is not a restrictive one, as all forms of vortex breakdown have been observed experimentally in the flows of liquids at low speed (Faler \& Leibovich 1977). The validity of the assumption of rotational symmetry has been discussed above. In a manner that is consistent with this assumption, the vortex axis is specified to be coincident with the pipe axis. The assumption of steady flow is questionable, since it is well documented that flows which experience bubble breakdown are not steady: unsteadiness is always observed within and downstream of the reversed flow region, and in the position of the breakdown structure (Faler \& Leibovich 1977). However, the search for equilibrium solutions seems valuable for two reasons: first, to shed more light on the calculation of stable, breakdown bubbles (Hafez et al. 1987), and secondly, to yield reasonable initial data for analysis involving time-integration of the governing equations.

The quasi-cylindrical equations are based on an additional assumption, which is directly analogous to the boundary-layer approximation in planar flows: viscous forces are sufficiently weak that the axial gradient of a flow variable is negligible relative to the radial gradient. Consequently, vortex evolution is assumed to occur 
on a much larger lengthscale than that defining the vortex core. The main implication of the quasi-cylindrical assumption is that the resulting equations are parabolic, where the streamwise direction is timelike.

\subsection{Governing equations}

Solutions of both the Navier-Stokes equations and the quasi-cylindrical equations for viscous, swirling flows through pipes were obtained in this work. The Navier-Stokes equations provide the basis for accurate simulations of vortex breakdown (within the limitations of the assumptions imposed), leading to predictions of breakdown position and structure. The quasi-cylindrical equations offer the potential of a much more efficient means by which breakdown can be predicted numerically. However, this efficiency is gained at the expense of great inaccuracy in the presence of reversed flow or large, axial gradients of flow quantities.

The governing equations corresponding to the two approaches described above are provided below in non-dimensional form. The radius of the vortex core at the inflow boundary, $\delta_{0}$, was chosen as a lengthscale, and the free-stream velocity, $W$, was chosen as a velocity scale. Henceforth, all variables will be assumed to be in nondimensional form.

Let $r$ and $z$ denote radial and axial position, and let $u, v$ and $w$ represent the radial, swirl and axial components, respectively. As the assumed flow is rotationally symmetric, flow properties depend only on $r$ and $z$. Thus, the equations governing the flow can be solved for a two-dimensional domain that represents a cross-section of the pipe flow.

\subsubsection{Navier-Stokes equations}

Under the assumptions of a steady, incompressible, rotationally symmetric flow, the Navier-Stokes equations are:

$$
\begin{gathered}
\psi_{z z}+\psi_{r r}-\frac{\psi_{r}}{r}=-r \eta \\
\frac{\psi_{r} \Gamma_{z}}{r}-\frac{\psi_{z} \Gamma_{r}}{r}=\frac{1}{\operatorname{Re}}\left(\Gamma_{z z}+\Gamma_{r r}-\frac{\Gamma_{r}}{r}\right) \\
\frac{\psi_{r} \eta_{z}}{r}-\frac{\psi_{z} \eta_{r}}{r}+\frac{\psi_{z} \eta}{r^{2}}=\frac{2 \Gamma \Gamma_{z}}{r^{3}}+\frac{1}{R e}\left(\eta_{z z}+\eta_{r r}+\frac{\eta_{r}}{r}-\frac{\eta}{r^{2}}\right),
\end{gathered}
$$

where $R e=W \delta_{0} / \nu$. Equations (1)-(3) are elliptic. The streamfunction, $\psi$, circulation (divided by $2 \pi$ ) $\Gamma$, and the azimuthal component of vorticity, $\eta$, are related to the velocity components as follows:

$$
\begin{aligned}
& u=-\frac{\psi_{z}}{r}, \quad w=\frac{\psi_{r}}{r}, \\
& \Gamma=r v, \quad \eta=u_{z}-w_{r} .
\end{aligned}
$$

\subsubsection{Quasi-cylindrical $(Q C)$ equations}

Under the assumptions of a steady, incompressible, rotationally symmetric flow, the $\mathrm{QC}$ equations are:

$$
\psi_{r r}-\frac{\psi_{r}}{r}=-r \eta
$$




$$
\begin{gathered}
\frac{\psi_{r} \Gamma_{z}}{r}-\frac{\psi_{z} \Gamma_{r}}{r}=\frac{1}{R e}\left(\Gamma_{r r}-\frac{\Gamma_{r}}{r}\right), \\
\frac{\psi_{r} \eta_{z}}{r}-\frac{\psi_{z} \eta_{r}}{r}+\frac{\psi_{z} \eta}{r^{2}}=\frac{2 \Gamma \Gamma_{z}}{r^{3}}+\frac{1}{R e}\left(\eta_{r r}+\frac{\eta_{r}}{r}-\frac{\eta}{r^{2}}\right),
\end{gathered}
$$

where

$$
\eta=w_{r}
$$

Equations $(6)-(8)$ are parabolic, with the streamwise direction serving as a timelike coordinate.

\subsection{Pipe description}

We analyse swirling flow through a two-stage cylindrical pipe of circular crosssection and total length $Z$. A schematic of the model is shown in figure 2 . The outlet was chosen to have the same cross-section area as the inlet, $\pi R_{0}^{2}$. The radius of the first stage was specified to vary sinusoidally over one spatial period, with a maximum radius equal to the inlet radius:

$$
R_{s}(z)=R_{0}+\alpha R_{0}[\cos (2 \pi z / L)-1] \quad(0 \leqslant z \leqslant L),
$$

where $L$ is the length of the first stage $(0<L<Z)$, and $\alpha$ is a parameter that determines the amount of contraction that occurs in the first stage. The ratio of the radius at the throat to the radius at the inlet is given by $1-2 \alpha$.

The second stage of the pipe was assumed to have a constant radius, $R_{0}$, so that

$$
R_{s}(z)=R_{0} \quad(z>L),
$$

We chose a uniform outlet section to make the specification of boundary conditions at the outflow boundary more accurate. Flows for more general second stages, such as divergent pipes, were not computed.

For almost all the calculations reported in this paper, the ratio $L / Z$ was fixed at $\frac{1}{3}$. Results were also obtained for a smaller ratio (i.e. a larger value of $Z$ with $R_{0}$ and $L$ fixed) to assess solution sensitivity to pipe length, as described in $§ 4$.

\subsection{Coordinate transformation}

Owing to the pipe contraction, a rectangular node arrangement cannot discretize the physical domain and simultaneously conform to the radial boundary of the pipe's first stage. To avoid a complicated and inaccurate treatment of the boundary conditions associated with the radial boundary, the equations of motion are solved over a rectangular computational domain, $\mathscr{R}$, that is discretized with a rectangular node arrangement. The computational domain is defined to extend over a portion of the $(\xi, \zeta)$-plane: $0 \leqslant \xi \leqslant Z$ and $0 \leqslant \zeta \leqslant R_{0}$. Mapping of points between the physical domain and the computational domain is achieved through the simple transformation

$$
\begin{gathered}
z=\xi \\
r=\zeta\left(\frac{R_{s}(z)}{R_{0}}\right) \rightarrow \zeta=r\left(\frac{R_{0}}{R_{s}(z)}\right) \equiv m r
\end{gathered}
$$

where

$$
m=m(z)=[1+\alpha(\cos (2 \pi z / L)-1)]^{-1} .
$$

Fist- and second-order derivatives of an arbitrary quantity, $f$, with respect to radial position are given by

$$
f_{r}=m f_{\zeta}, \quad f_{r r}=m^{2} f_{\zeta \zeta}
$$


while first- and second-order derivatives of $f$ with respect to axial position are given by

$$
\begin{gathered}
f_{z}=f_{\xi}+\dot{m} r f_{\zeta} \\
f_{z z}=f_{\xi \xi}+(\dot{m} r)^{2} f_{\zeta \zeta}+2 \dot{m} r f_{\xi \zeta}+\dot{m} r f_{\zeta} .
\end{gathered}
$$

Values of $\dot{m}$ and $\ddot{m}$ are obtained by differentiating (14).

Following transformation, the Navier-Stokes equations are written as

$$
\begin{gathered}
\psi_{\xi \xi}+\left(m^{2}+\dot{m}^{2} r^{2}\right) \psi_{\zeta \xi}+2 \dot{m} r \psi_{\xi \zeta}+\left(\ddot{m} r-\frac{m}{r}\right) \psi_{\zeta}+r \eta=0 \\
\frac{m}{r}\left(\psi_{\zeta} \Gamma_{\xi}-\psi_{\xi} \Gamma_{\zeta}\right)-\frac{1}{\operatorname{Re}}\left[\Gamma_{\xi \xi}+\left(m^{2}+\dot{m}^{2} r^{2}\right) \Gamma_{\zeta \zeta}+2 \dot{m} r \Gamma_{\xi \zeta}+\left(\ddot{m} r-\frac{m}{r}\right) \Gamma_{\zeta}\right]=0 \\
\frac{m}{r}\left(\psi_{\zeta} \eta_{\xi}-\psi_{\xi} \eta_{\zeta}\right)+\frac{\eta}{r^{2}}\left(\psi_{\xi}+\dot{m} r \psi_{\zeta}\right)-\frac{2 \Gamma^{3}}{r^{3}}\left(\Gamma_{\xi}+\dot{m} r \Gamma_{\zeta}\right) \\
-\frac{1}{R e}\left[\eta_{\xi \xi}+\left(m^{2}+\dot{m}^{2} r^{2}\right) \eta_{\zeta \zeta}+2 \dot{m} r \eta_{\zeta \zeta}+\left(\ddot{m} r+\frac{m}{r}\right) \eta_{\zeta}-\frac{\eta}{r^{2}}\right]=0
\end{gathered}
$$

The transformed form of the QC equations is provided in the Appendix.

\subsection{Boundary conditions}

The boundary conditions placed on the Navier-Stokes equations are slightly different from those used with the QC equations. Specification of conditions on $\psi, \eta$ and $\Gamma$ on the four boundaries of the computational domain, $\mathscr{R}$, is required for the Navier-Stokes equations. Enforcement of conditions on these same variables on the outflow boundary of $\mathscr{R}$ is not required for the QC equations.

\subsubsection{Inflow boundary (S1)}

On the inflow boundary, the streamfunction and circulation profiles are specified to have the following forms:

$$
\psi(0, r)=\frac{1}{2} r^{2}, \quad \Gamma(0, r)=V\left(1-\exp \left[-r^{2}\right]\right)
$$

which are equivalent to the axial and azimuthal velocity profiles

$$
w(0, r)=1, \quad v(0, r)=\frac{V}{r}\left(1-\exp \left[-r^{2}\right]\right)
$$

The azimuthal velocity profile provides solid-body rotation in the vortex core $(v \sim r)$, and irrotational flow $(v \sim 1 / r)$ outside the core. The 'vortex strength', $V$, is approximately equal to the circulation, $\Gamma$, of the vortex. In cases of interest, the peak swirl velocity is of the same order of magnitude as the free-stream axial velocity. The effect of a velocity excess or defect in the vortex core was not investigated in this work.

The inflow condition on vorticity is complicated by the dependence of vorticity on the axial gradient of $u$, which is not generally known at the upstream boundary. This problem has been dealt with in two ways for straight pipes by previous investigators for the case of $w(0, r)=1$. Krause et al. (1983) enforced a Dirichlet condition, $\eta(r)=0$, on vorticity at the inflow boundary. This condition implies that $u_{z}$ and $w_{z z}$ vanish along the inflow boundary, but allows the local flow state to possess an axial gradient 
in the axial velocity. This is a reasonable condition for a viscous vortex that is slowly decaying as it enters the test section represented by the computational domain (this condition is automatically enforced through application of the $\mathrm{QC}$ equations). Krause et al. obtained steady-state solutions to the time-dependent form of (1)-(3) in the absence of reversed axial flow, but were unable to find steady-state solutions with reversed flow. Hafez et al. (1987) applied an implicit condition on inflow vorticity,

$$
\eta(0, r)=-\frac{1}{r} \psi_{z z}(0, r)
$$

which follows from (1) and (22). $\psi_{z z}(0, r)$ was estimated assuming $u(0, r)=0$. Steadystate solutions with reversed flow were obtained by enforcing this condition. Grabowski (1974) solved the Navier-Stokes equations in velocity-pressure form, and also obtained steady-state solutions with reversed flow by directly enforcing $u(0, r)=0$.

Results using both types of inflow conditions on vorticity have been compared by Beran (1989). Results do not differ significantly unless reversed flow develops, consistent with the observations described above. With the onset of reversed flow, large axial gradients in flow quantities are observed in the vicinity of the inflow boundary so that there is an increased sensitivity to the inflow conditions that are enforced. With reversed flow in a straight pipe, the Dirichlet condition on vorticity is no longer physically sensible, since there is not a rational justification for $u_{z}(0, r)=0$. However, when the pipe geometry is modified in the manner treated in this investigation, vortex breakdown is prevented from occurring near the inflow boundary by the adverse pressure gradient that develops downstream of the pipe throat. With breakdown separated from the inflow boundary, large axial derivatives do not take place near that boundary, so that the Dirichlet condition remains acceptable. Consequently the Dirichlet condition,

$$
\eta(0, \zeta)=0
$$

is enforced in this investigation. (Assuming, along the inlet, that $w=1$ and the axial derivative of radial velocity is negligible, $u_{z} \approx 0$, is reasonable, following consideration of $(5 b)$, to set $\eta=0$ as an inlet boundary condition.)

\subsubsection{Outflow boundary (S3)}

At the outflow boundary, the flow is assumed to be governed by the QC equations, implying that streamwise derivatives associated with viscous dissipation are negligible:

$$
\psi_{\xi \xi}(Z, \zeta) \approx 0, \quad \Gamma_{\xi \xi}(Z, \zeta) \approx 0, \quad \eta_{\xi \xi}(Z, \zeta) \approx 0
$$

Along S3, streamwise derivatives contained in (7) and (8) are approximated with first-order accurate upwind-difference expressions.

\subsubsection{Axis of symmetry (S4)}

The axis of symmetry is a streamline along which the radial and azimuthal velocity components vanish, and on which the axial velocity experiences a local extremum with respect to radial position. Consequently, we can set

$$
\psi(\xi, 0)=\Gamma(\xi, 0)=\eta(\xi, 0)=0
$$




\subsubsection{Pipe wall (S2)}

The flow is assumed to be inviscid at the pipe wall, so that impermeability, but not no-slip, is enforced at this boundary. Thus, the wall is a stream surface:

$$
\psi\left(\xi, R_{0}\right)=\frac{1}{2} R_{0}^{2}
$$

Without friction, the circulation equation reduces to (on the pipe wall)

$$
\frac{m}{R_{s}}\left(\psi_{\zeta} \Gamma_{\xi}-\psi_{\xi} \Gamma_{\zeta}\right)=0
$$

which implies (an expression of Kelvin's theorem):

$$
\Gamma\left(\xi, R_{0}\right)=V\left(1-\exp \left[-R_{0}^{2}\right]\right)
$$

In a similar fashion, the vorticity equation reduces to

$$
\frac{m \eta_{\xi}}{R_{s}} \psi_{\zeta}+\frac{\eta \dot{m}}{R_{s}} \psi_{\zeta}-\frac{2 \Gamma \dot{m}}{R_{s}^{2}} \Gamma_{\zeta}=0, \quad\left(m \eta_{\xi}+\eta \dot{m}\right) \psi_{\zeta}-\frac{2 \Gamma \dot{m}}{R_{s}} \Gamma_{\zeta}=0
$$

In the vicinity of the radial boundary, $\Gamma$ is assumed to be a function of $\psi$ only, leading to

$$
\Gamma=\Gamma(\psi)=V(1-\exp [-2 \psi]) \quad(\text { near the pipe wall })
$$

With (29), and the assumption that $\psi_{\zeta}$ does not vanish in the vicinity of the radial boundary, (28) can be written as

$$
\frac{\partial}{\partial \xi}(m \eta)-\frac{\Gamma \dot{\Gamma}}{R_{0}} \frac{\mathrm{d}}{\mathrm{d} \xi}\left(m^{2}\right)=0
$$

where $\dot{\Gamma} \equiv \mathrm{d} \Gamma / \mathrm{d} \psi$, Integration of $(30)$ with respect to $\xi$ along the pipe wall, using $\eta\left(0, R_{s}\right)=0$ and $m(0)=1$, yields

$$
\eta\left(\xi, R_{0}\right)=\frac{\Gamma \dot{\Gamma}}{R_{0}}\left(m-\frac{1}{m}\right)
$$

Together, (29) and (31) provide a Dirichlet condition on azimuthal vorticity along the radial boundary:

$$
\eta\left(\xi, R_{0}\right)=\frac{2 V^{2}}{R_{0}}\left(1-\exp \left[-R_{0}^{2}\right]\right) \exp \left[-R_{0}^{2}\right]\left(m-\frac{1}{m}\right) .
$$

Allowance for slip at the pipe wall will prevent rigorous comparison between results obtained with the analysis described here and experimental data, owing to the absence of a wall boundary layer. Such a structure can have a significant impact on breakdown behaviour if boundary-layer thickness is sufficiently large (Sarpkaya 1974). While we see modelling of boundary-layer-vortex interaction to be important for predicting breakdown location and other practical quantities, we do not consider it to be especially important in describing the genesis of vortex breakdown. In the specified absence of natural, three-dimensional asymmetries, disregard for the wall boundary layer may be a less important restriction.

\section{Numerical procedure}

The numerical procedure by which equilibrium solutions of the Navier-Stokes are obtained is described in this section. A different procedure is used for the QC equations and this technique is described in the Appendix. 


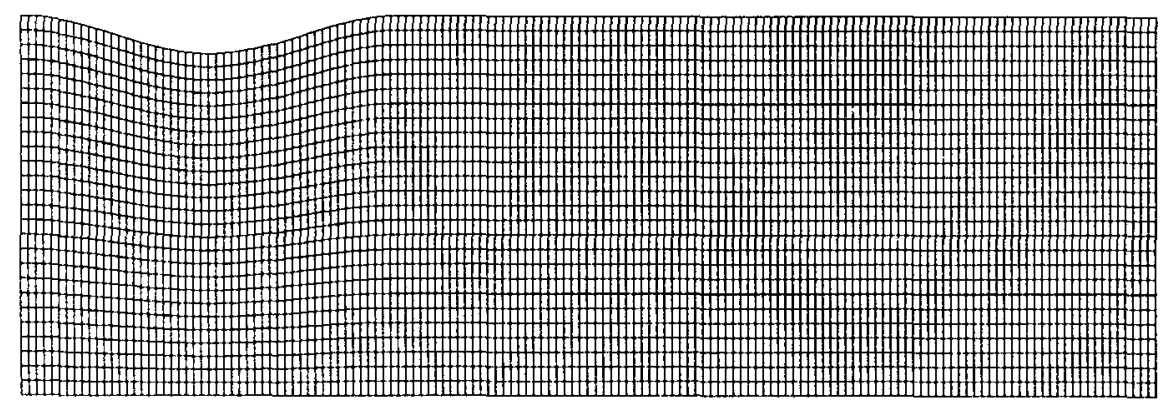

FIgure 3. A typical grid (G2) with $\alpha=0.05$.

\begin{tabular}{llllllr} 
Grid & \multicolumn{1}{c}{$\alpha$} & $R_{0}$ & $Z$ & $J$ & $I$ & \multicolumn{1}{c}{$I_{\mathrm{L}}$} \\
G1 & 0.05 & 2 & 30 & 27 & 151 & 51 \\
G2 & 0.05 & 2 & 30 & 40 & 151 & 51 \\
G3 & 0.05 & 2 & 30 & 27 & 301 & 101 \\
G4 & 0.025 & 2 & 30 & 27 & 301 & 101 \\
G5 & 0.0 & 2 & 30 & 27 & 301 & 101 \\
G6 & 0.05 & 2 & 30 & 40 & 301 & 101 \\
G7 & 0.05 & 3 & 30 & 40 & 301 & 101 \\
G8 & 0.05 & 2 & 60 & 27 & 601 & 101 \\
G9 & 0.05 & 2 & 120 & 14 & 1201 & 101
\end{tabular}

TABLE 1. Grids used in the calculation of solutions of the Navier-Stokes equations

\begin{tabular}{lllrrrc} 
Grid & \multicolumn{1}{c}{$\propto$} & $R_{0}$ & $Z$ & $J$ & $I$ & $I_{\mathbf{L}}$ \\
G10 & 0.05 & 2 & 30 & 27 & 901 & 301 \\
G11 & 0.025 & 2 & 30 & 27 & 901 & 301 \\
G12 & 0.0 & 2 & 30 & 27 & 901 & 301 \\
G13 & 0.05 & 2 & 120 & 14 & 3601 & 301
\end{tabular}

TABLE 2. Grids used in the calculation of solutions of the QC equations

\subsection{Grid description}

Solutions were obtained with several different grids. The characteristic features of the different grids used in this study are shown in tables 1 and 2 . Solutions of the Navier-Stokes equations were obtained with grids listed in table 1, while solutions of the AC equations were obtained with grids listed in table 2. A typical grid (G1) is shown in figure 3. Each grid was constructed as follows (see figure 4 for a schematic of the node arrangement).

(i) The node index in the axial direction, $i$, satisfied $1 \leqslant i \leqslant I$, where $I$ is a specified constant.

(ii) The node index in the radial direction, $j$ satisfied $1 \leqslant j \leqslant J$, where $J$ is a specified constant.

(iii) The node index $i$ satisfied $1 \leqslant i \leqslant I_{\mathrm{L}}$ for the first stage of the pipe.

(iv) The node spacing in the axial direction, $\Delta_{\xi}$, was specified to be uniform, i.e. $\Delta_{\varepsilon}=Z /(I-1)$ throughout the computational domain.

(v) The node spacing in the radial direction, $\Delta_{\zeta}$, was specified to be uniform, i.e. $\Delta_{\zeta}=R_{0} /(J-1)$ throughout the computational domain. 


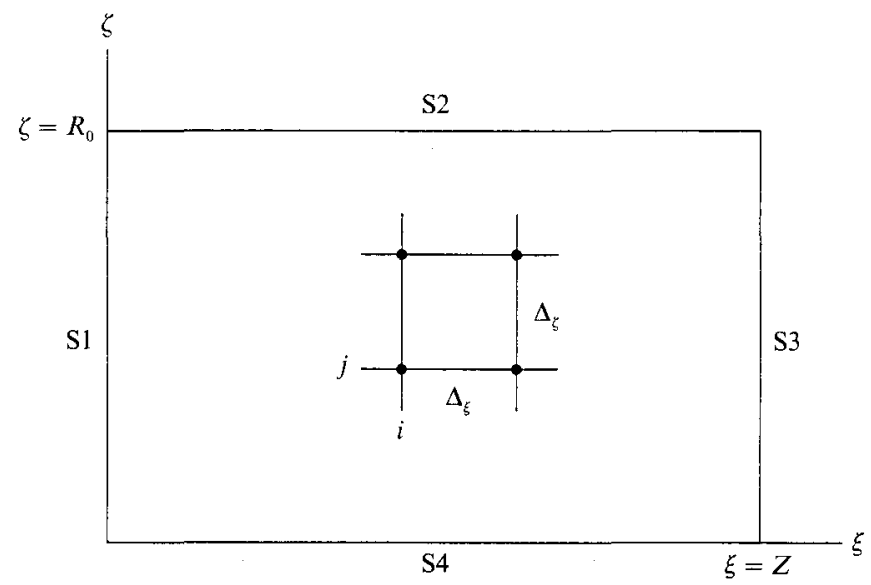

FIGURE 4. Schematic of the node arrangement within the computational domain, $\mathscr{R}$.

\subsection{Discretization of equations}

The unknown values of $(\psi, \Gamma, \eta)$ at each node point are collocated into a single vector, $\boldsymbol{x}$, in the following order:

$$
x=\left(\psi_{1}, \Gamma_{1}, \eta_{1}, \psi_{2}, \Gamma_{2}, \eta_{2}, \ldots, \psi_{k}, \Gamma_{k}, \eta_{k}, \ldots, \psi_{N}, \Gamma_{N}, \eta_{N}\right)^{T}
$$

where $k=(i-1) J+j, \quad N=I J$.

At nodes interior to $\mathscr{R}$, derivatives appearing in the Navier-Stokes equations are approximated with second-order accurate, central-difference operators. The outflow conditions are the $\mathrm{QC}$ equations in discrete form. Streamwise derivatives are approximated with one-sided, first-order-accurate expressions :

$$
\Delta_{\xi} \Gamma_{\xi} \doteq \Gamma_{I}-\Gamma_{I-1}, \quad \Delta_{\xi} \psi_{\xi} \doteq \psi_{I}-\psi_{I-1} \quad(1<j<J) .
$$

Discrete equations are collocated into an array of equations, $F$, organized in the same way as $\boldsymbol{x}$. For a particular mesh geometry, $R e, V$ and $\alpha$ are free parameters of the discrete Navier-Stokes model. The dependence of $F$ on these parameters and the solution vector, $\boldsymbol{x}$, is represented in the set of discrete equations

$$
F(x ; R e, V, \alpha)=0 .
$$

\subsection{Solution of equations}

The system of nonlinear algebraic equations (34) is solved through the combined use of Newton's method and the pseudo-arclength continuation method. We consider systems of equations of the form

$$
F(x ; \lambda)=0,
$$

where $\boldsymbol{F}$ is a set of $N$ equations dependent on $\boldsymbol{x}$ and $\lambda, \boldsymbol{x}$ is an $N$-dimensional vector of unknowns, and $\lambda$ is a free parameter. (In this study, the vortex strength, $V$, is a free parameter that is varied, while the parameters $R e$ and $\alpha$ are held fixed.) Newton's method is an algorithm to compute $\boldsymbol{x}$ for a specified value of $\lambda$. The pseudoarclength continuation method, like other continuation methods, is an algorithm that uses information about $\boldsymbol{F}$ and $\boldsymbol{x}$ to compute another solution corresponding to a different value of $\lambda$. 


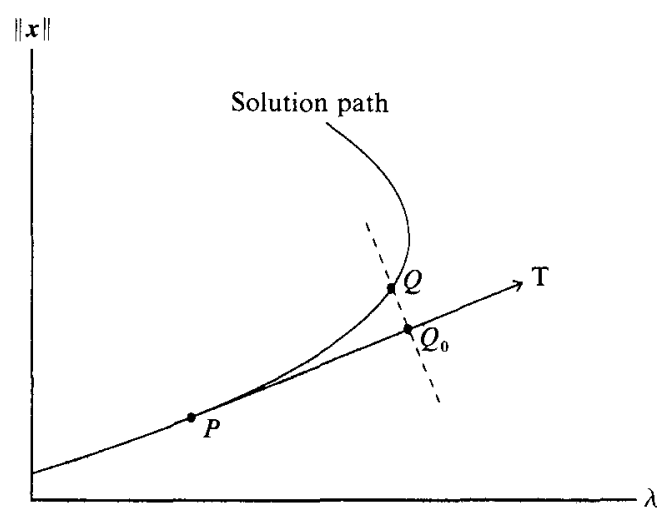

FIGURE 5. Graphical representation of the solution procedure using the pseudo-arclength continuation method.

The method used to solve the discrete form of the QC equations is described in the Appendix.

When used with continuation, Newton's method is a robust algorithm for computing solutions to systems of equations such as (35). The algorithm is iterative. Given $\lambda$ and an initial approximation to the solution vector, $\boldsymbol{x}^{n}$, one Newton iterate consists of the computation of an improved approximation, $\boldsymbol{x}^{n+1}$, obtained by solving the linear system of equations,

$$
\boldsymbol{F}_{\boldsymbol{x}}\left(\boldsymbol{x}^{n} ; \lambda\right)\left(\boldsymbol{x}^{n+1}-\boldsymbol{x}^{n}\right)=-\boldsymbol{F}\left(\boldsymbol{x}^{n} ; \lambda\right)=-\boldsymbol{F}^{n} .
$$

Successive Newton iterates were computed until the weighted L2 norm of the residuals,

$$
\left\|F^{n}\right\| \equiv\left(\sum_{k=1}^{N} \Delta_{z} \Delta_{r} F_{k}^{2}\left(x^{n} ; \lambda\right)\right)^{\frac{1}{2}}
$$

became less than $5 \times 10^{-5}$.

The linear operator in (36) is the Jacobian matrix, the elements of which are given by

$$
F_{x} \equiv\left[\frac{\partial F_{i}}{\partial x_{j}}\right]
$$

In this study, Jacobian matrices were computed by evaluating analytically derived expressions for matrix elements at the current solution estimate. If the Jacobian matrix is nonsingular at the solution, $x^{*}$, and if the initial guess, $x^{n}$, is sufficiently good, then Newton's method is guaranteed to converge with a quadratic convergence rate. The systems of linear equations encountered with each Newton iterate were solved to machine precision by Gaussian elimination with partial pivoting. The Jacobian matrix was defined to be singular if in the process of solving (36), the maximum pivot had a magnitude of less than about 100 times machine precision. No singular matrices were encountered in this study.

The continuation method used in this study to compute successive solution points on the solution path $\boldsymbol{x}(\lambda)$ was Keller's (1977) pseudo-arclength continuation method. To facilitate the description of Keller's method, refer to figure 5, which shows the solution path as it would appear when the norm of the solution vector is plotted against $\lambda$. 
The arclength, $s$, is chosen to parameterize the path, so that on the path, $\boldsymbol{x}=\boldsymbol{x}(s)$, $\lambda=\lambda(s)$ and $\boldsymbol{F}=\boldsymbol{F}(s)=0$. The continuation process begins with the computation of the vector tangent to the path at a known solution point. From

$$
\frac{\mathrm{d}}{\mathrm{d} s} \boldsymbol{F}(\boldsymbol{x}(s) ; \lambda(s))=0
$$

and the chain-rule

$$
\boldsymbol{F}_{\boldsymbol{x}}(s) \dot{\boldsymbol{x}}(s)+\boldsymbol{F}_{\lambda}(s) \dot{\lambda}(s)=\mathbf{0}
$$

where

$$
\dot{\boldsymbol{x}}(s) \equiv \frac{\mathrm{d} x}{\mathrm{~d} s}(s), \quad \dot{\lambda}(s) \equiv \frac{\mathrm{d} y}{\mathrm{~d} s}(s), \quad \boldsymbol{F}_{\lambda} \equiv \frac{\partial \boldsymbol{F}}{\partial \lambda} .
$$

The definition of arclength is

$$
\dot{\boldsymbol{x}}(s) \cdot \dot{\boldsymbol{x}}(s)+\dot{\lambda}^{2}(s)=1 .
$$

Conditions (38) and (40) are solved for the tangent vector,

$$
\boldsymbol{T}(s) \equiv\left(\begin{array}{c}
\dot{\boldsymbol{x}}(s) \\
\dot{\lambda}(s)
\end{array}\right)
$$

at points on the solution path for which the Jacobian matrix is non-singular:

$$
\begin{gathered}
\dot{\lambda}(\mathrm{s})= \pm 1 /(1+\boldsymbol{\Phi} \cdot \boldsymbol{\Phi})^{\frac{1}{2}}, \\
\dot{\boldsymbol{x}}(s)=-\dot{\lambda}(s) \boldsymbol{\Phi}, \\
\boldsymbol{\Phi} \equiv \boldsymbol{F}_{\boldsymbol{x}}^{-1}(s) \boldsymbol{F}_{\lambda}(s) .
\end{gathered}
$$

The sign in (42) is indeterminate because the tangent can point in either the direction of increasing $s$ or decreasing $s$.

Given a known solution point, $P$, a neighbouring solution point, $Q$, is required to lie on a planar manifold perpendicular to the path tangent projected from the known solution point (see figure 5). The parameter $d$ fixes the distance between the point $P$ and the intersection point, $Q_{0}$, of the path tangent and the planar manifold perpendicular to the tangent. Thus,

$$
\left(\begin{array}{l}
x \\
\lambda
\end{array}\right)_{Q_{0}}=\left(\begin{array}{l}
x \\
\lambda
\end{array}\right)_{P}+d\left(\begin{array}{l}
\dot{\boldsymbol{x}} \\
\dot{l}
\end{array}\right)_{P} .
$$

The condition that $Q$ lie on a planar manifold perpendicular to the path tangent at $Q_{0}$ is

$$
\dot{D}\left(x_{Q} ; \lambda_{Q}\right) \equiv \dot{x}_{P} \cdot\left(x_{Q}-x_{P}\right)+\left(\lambda_{Q}-\lambda_{P}\right) \dot{\lambda}_{P}=d,
$$

and is added to (35) to form an enlarged system of nonlinear equations. The solution $Q$ is computed through Newton iteration until a converged solution to $F=0$ and $D=d$ is found. $Q_{0}$ is used as an initial approximation. With the computation of $Q$, additional points on the path are found by repeating the process outlined above.

\subsection{Diagnostics}

Axial velocity on the centreline, $w_{\mathrm{c}}(z) \equiv w(z, 0)$, is a good measure of the behaviour of the core flow and gives a direct indication of where vortex breakdown occurs. Furthermore, the quantity

$$
Q \equiv \min _{z}\left[w_{\mathrm{e}}(z)\right]
$$

was used to represent computed solutions in the presentation of solution paths. 
At a particular axial station, $w_{c}$ is computed using the relationship

$$
w_{\mathrm{e}}=\lim _{r \rightarrow 0}\left(\frac{1}{r} \psi_{r}\right)
$$

For $w_{\mathrm{c}}$ to remain bounded on the centreline, we must have $\psi_{r}(z, 0)=0$, and consequently

$$
w_{\mathrm{c}}=\left.\psi_{r r}\right|_{r=0} .
$$

$w_{c}(z)$ is computed to second-order accuracy through examination of two Taylor series expansions of $\psi$ evaluated at $r=0$ :

$$
\psi_{i, 2}=\frac{1}{2}\left(w_{\mathrm{c}}\right)_{\mathrm{i}} r_{i, 2}^{2}+\frac{1}{6} \psi_{r r} r_{i, 2}^{3}+O\left[r_{i, 2}^{4}\right], \quad \psi_{i, 3}=\frac{1}{2}\left(w_{\mathrm{c}}\right)_{\mathrm{i}} r_{i, 3}^{2}+\frac{1}{6} \psi_{r r r} r_{i, 3}^{3}+O\left[r_{i, 3}^{4}\right] .
$$

And, since $r_{i, 3}=2 r_{i, 2}$,

$$
\psi_{i, 3}=2\left(w_{\mathrm{c}}\right)_{\mathrm{i}} r_{i, 2}^{2}+\frac{8}{6} \psi_{r r r} r_{i, 2}^{3}+O\left[r_{i, 2}^{4}\right]
$$

Equations (48a and (49) yield

$$
\left(w_{c}\right)_{i}=\frac{1}{r_{i, 2}^{2}}\left(4 \psi_{i, 2}-\frac{1}{2} \psi_{i, 3}\right)=\frac{1}{\Delta_{r}^{2}}\left(4 \psi_{i, 2}-\frac{1}{2} \psi_{i, 3}\right) .
$$

\section{Results}

Results were obtained for several different values of the vortex strength, $V$, the Reynolds number, $R e$, the constriction parameter, $\alpha$, the axial node spacing, $\Delta_{\xi}$, and the radial node spacing, $\Delta_{\zeta}$. The length of the first and second stages of the pipe were held constant at 10 and 20 , respectively, and the inlet radius, $R_{0}$, was held constant at 2 (the physical domain defined by this choice of $Z$ and $R_{0}$ will be referred to as the 'standard' domain). Solutions of the Navier-Stokes equations were calculated with the pseudo-arclength continuation method using $V$ as a free parameter. This process was repeated for

four values of $R e: 250,500,1000$, and 2000 (with $I=301$ and $J=27$ ); three values of $\alpha: 0.0,0.025$, and 0.05 (with $I=301$ and $J=27$ );

two values of $I: 151$ and 301 (with $\alpha=0.05$ and $R e=250,500,1000$ and 2000 ); two values of $K: 27$ and 40 (with $\alpha=0.05$ and $R e=250,500,1000$ and 2000).

In addition, four solution paths were computed for each of three modified domains:

an elongated pipe (G8) to assess solution sensitivity to the parameter $Z$;

a widened pipe (G7) to assess solution sensitivity to the parameter $R_{0}$;

a very elongated pipe (G9) to observe solution behaviour at large Reynolds numbers.

Calculations were carried out on Stardent GS-2000 and Cray-YMP supercomputers. Solutions of the QC equations were also obtained, following the procedure outlined in the Appendix, to correlate with the solutions of the Navier-Stokes equations. The QC equations were integrated using grids G10, G11 and G12 for which $I=901$ and grid G13 for which $I=3601$.

A very small axial node spacing was found necessary to compensate for the firstorder accuracy of the integration scheme.

\subsection{Procedure}

Solution paths of the Navier-Stokes equations were calculated through the following procedure: 
(i) for a specified Reynolds number, a starting solution point was computed at a relatively small value of the circulation (where solution uniqueness was assumed) using as an initial guess

$$
\psi(\xi, \zeta)=\frac{1}{2} \zeta^{2}, \quad \Gamma(\xi, \zeta)=V\left(1-\exp \left[-\zeta^{2}\right]\right), \quad \eta(\xi, \zeta)=0
$$

(ii) the pseudo-arclength continuation method was employed to compute additional points on the solution path using the starting point;

(iii) continuation was performed until a sufficient number of points had been computed or until Newton's method failed to converge to a point on the solution path.

Several of the computed paths are well represented by the solution diagram illustrated in figure 1 (e.g. see figure $15 b$ ). On the typical path, the continuation method traced, in the direction of increasing $V$, the uppermost branch of solution points, for which reversed flow did not occur. As can be seen in figure 1, this branch ends in a limit point (termed the 'primary' limit point by the authors, since it is the first limit point encountered during continuation), upon which the path folds back on itself. Following passage through the primary limit point, the continuation method traced the path in the direction of decreasing $V$, leading eventually to the development of reversed flow and the formation of a second limit point (the 'secondary' limit point). At large Reynolds numbers, secondary limit points were sometimes not calculated owing to the inability of the continuation method to converge to points on the solution path.

We considered the continuation method to have 'failed' when more than five Newton iterates were required to satisfy the convergence criterion, $\|\boldsymbol{F}\|<5 \times 10^{-5}$. Invariably, convergence was slower when the solution was in the vicinity of a limit point. For such solutions, if the chosen length, $d$, was too large, then continuation would fail. When it was clear that the solution was smooth and that failure was due to a poor choice of $d$, then the value of $d$ was reduced and the continuation process reattempted. Convergence to spurious solutions that lacked smoothness was also very poor. Non-smooth solutions were characterized by high-frequency, spatial oscillations of vortex properties (in the axial direction), referred to as 'wiggles' by Anderson, Tannehill \& Pletcher (1984). These dispersive errors were most severe between the inflow boundary and the axial station at which a minimum was achieved $z_{Q}$, and were only evident when the mesh Reynolds number (defined here as $R e \Delta_{\xi}$ ) was sufficiently large. In addition, the appearance of numerical oscillations was highly dependent on the circulation, since they were only observed when the flow structure in the neighbourhood of $z=z_{Q}$ was not well resolved by the grid (i.e. when the flow had experienced, or nearly experienced, breakdown). The development of numerical oscillations, and an associated inability to precisely locate breakdown structures owing to grid coarseness, led to non-smooth behaviour of the solution path, as is illustrated in figure 8 for $I=151$ and $R e=500$. On such path segments, numerous limit points were computed, convergence was poor and the continuation method would usually fail. Grid refinement, through reduction in axial node spacing, was found to eliminate some, but not all, of the spurious behaviour. This is described further below when solution sensitivity to node spacing is assessed.

At selected Reynolds numbers, solutions of the QC equations were computed at successively larger circulations until it become evident that the integration process had 'failed'. The authors observed two types of failure. In the first failure mode, radial velocity grows dramatically over a very short axial distance, leading to numerical divergence of the integration. In the second mode, the behaviour of the 


\begin{tabular}{cccrccc} 
Solution path & Grid & \multicolumn{1}{c}{$\alpha$} & \multicolumn{1}{c}{$R e$} & Path range $(V)$ & $V_{1 \mathrm{p}}$ & $V_{\mathrm{c}}$ \\
SP1 & G3 & 0.050 & 250 & $1.20-1.6146$ & Absent & 1.429 \\
SP2 & G4 & 0.025 & 250 & $1.20-1.6337$ & Absent & 1.470 \\
SP3 & G5 & 0.0 & 250 & $1.20-1.6074$ & 1.6209 & $\mathbf{1 . 5 6 5}$ \\
SP4 & G3 & 0.050 & 500 & $1.20-1.5724$ & 1.4813 & 1.459 \\
SP5 & G4 & 0.025 & 500 & $1.20-1.5631$ & 1.5132 & 1.486 \\
SP6 & G5 & 0.0 & 500 & $1.20-1.5500$ & 1.6044 & 1.565 \\
SP7 & G3 & 0.050 & 1000 & $1.20-1.5725$ & 1.5069 & 1.514 \\
SP8 & G4 & 0.025 & 1000 & $1.20-1.4956$ & 1.5299 & 1.530 \\
SP9 & G5 & 0.0 & 1000 & $1.20-1.5196$ & 1.5989 & 1.566 \\
SP10 & G3 & 0.050 & 2000 & $1.20-1.4731$ & 1.5419 & 1.557 \\
SP11 & G4 & 0.025 & 2000 & $1.20-1.4847$ & 1.5564 & 1.558 \\
SP12 & G5 & 0.0 & 2000 & $1.20-1.4529$ & 1.5591 & 1.567 \\
SP13 & G1 & 0.050 & 250 & $1.45-1.5559$ & Absent & - \\
SP14 & G1 & 0.050 & 500 & $1.45-1.5085$ & 1.4816 & - \\
SP15 & G1 & 0.050 & 1000 & $1.45-1.4832$ & 1.5071 & - \\
SP16 & G1 & 0.050 & 2000 & $1.45-1.5103$ & 1.5417 & - \\
SP17 & G2 & 0.050 & 250 & $1.45-1.5649$ & Absent & - \\
SP18 & G2 & 0.050 & 500 & $1.45-1.4752$ & 1.4821 & - \\
SP19 & G2 & 0.050 & 1000 & $1.45-1.4847$ & 1.5078 & - \\
SP20 & G2 & 0.050 & 2000 & $1.45-1.5113$ & 1.5426 & - \\
SP21 & G6 & 0.050 & 250 & $1.45-1.5296$ & Absent & - \\
SP22 & G6 & 0.050 & 500 & $1.45-1.4738$ & 1.4818 & - \\
SP23 & G6 & 0.050 & 1000 & $1.45-1.4729$ & 1.5077 & - \\
SP24 & G6 & 0.050 & 2000 & $1.45-1.5245$ & 1.5427 & - \\
GE & & & & &
\end{tabular}

TABLE 3. Solution paths computed by varying vortex strength (standard domain)

\begin{tabular}{cccrccc} 
Solution path & Grid & $\alpha$ & \multicolumn{1}{c}{$R e$} & Path range $(V)$ & $V_{\text {lp }}$ & $V_{\mathrm{c}}$ \\
SP25 & G8 & 0.050 & 250 & $1.20-\mathbf{1 . 5 9 6 8}$ & Absent & - \\
SP26 & G8 & 0.050 & 500 & $1.20-\mathbf{1 . 5 6 6 2}$ & 1.4808 & - \\
SP27 & G8 & 0.050 & 1000 & $1.45-\mathbf{1 . 5 5 3 0}$ & 1.5068 & - \\
SP28 & G8 & 0.050 & 2000 & $1.45-\mathbf{1 . 4 8 3 1}$ & 1.5419 & - \\
SP29 & G7 & 0.050 & 250 & $1.20-1.5685$ & Absent & - \\
SP30 & G7 & 0.050 & 500 & $1.20-1.6506$ & 1.4818 & - \\
SP31 & G7 & 0.050 & 1000 & $1.20-1.5569$ & 1.5075 & - \\
SP32 & G7 & 0.050 & 2000 & $1.20-1.5379$ & 1.5427 & - \\
SP33 & G9 & 0.050 & 6000 & $0.00-1.5348$ & 1.5739 & 1.580 \\
SP34 & G9 & 0.050 & 5000 & $0.00-1.5293$ & 1.5698 & 1.579 \\
SP35 & G9 & 0.050 & 4000 & $0.00-1.5230$ & 1.5639 & 1.577 \\
SP36 & G9 & 0.050 & 3000 & $1.20-1.5131$ & 1.5542 & 1.575
\end{tabular}

TABLE 4. Solution paths computed by varying vortex strength (modified domains)

computed solution changes over a similarly small axial distance in a way that is nonphysical. This anomalous behaviour, observed by Beran (1989), does not involve large values of $u(\zeta)$ or numerical divergence.

\subsection{Tabulated results}

The parameter values used in the calculation of solution paths of the Navier-Stokes equations are summarized in table 3 (standard domain) and table 4 (modified domains). Also included are the locations of primary limit points, $V_{\text {lp }}$, on the solution paths, the starting and end points of the paths, and the critical value of $V$ beyond which the QC equations fail, $V_{\mathrm{c}}$. 

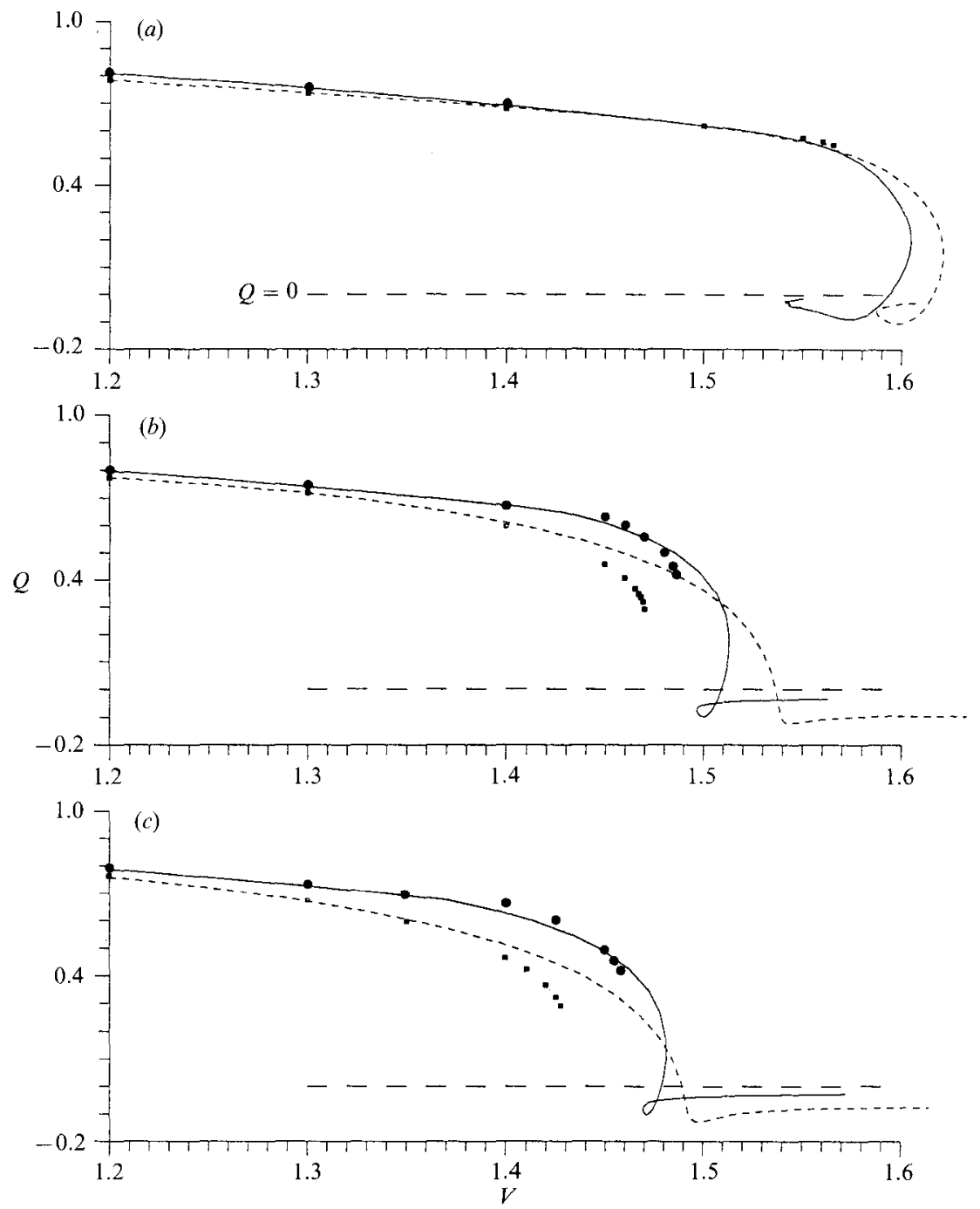

Figure 6. Solution diagrams for the Navier-Stokes equations with ---, Re $=250$ and $R e=500$ and the QC equations with $\square, R e=250$ and $\bigcirc, R e=500:(a) \alpha=0.0 ;(b) \alpha=0.025$; (c) $\alpha=0.05$.

The authors did not find any evidence to indicate that the starting points of paths were non-unique. However, the potential for solution multiplicity at the starting values of $V$ cannot be ruled out based on the results of this study. As one check of the assumption of uniqueness, paths SP33-SP35 were started at $V=0$, where it is reasonable to expect solutions to be unique. Points on these three paths for non-zero values of $V$ were identical (up to the primary limit points) to those obtained by starting the continuation process at the same values of $V$. Also, continuation in $R e$ did not reveal solution branches different to those collectively implied by paths SP1-SP36.

\subsection{Continuation runs}

The solution paths SP1-SP12 are presented in figures 6 and 7 as represented by $Q$, the minimum value of the centreline axial velocity. Solutions of the QC equations at selected values of the vortex strength are also included. By definition, breakdown 

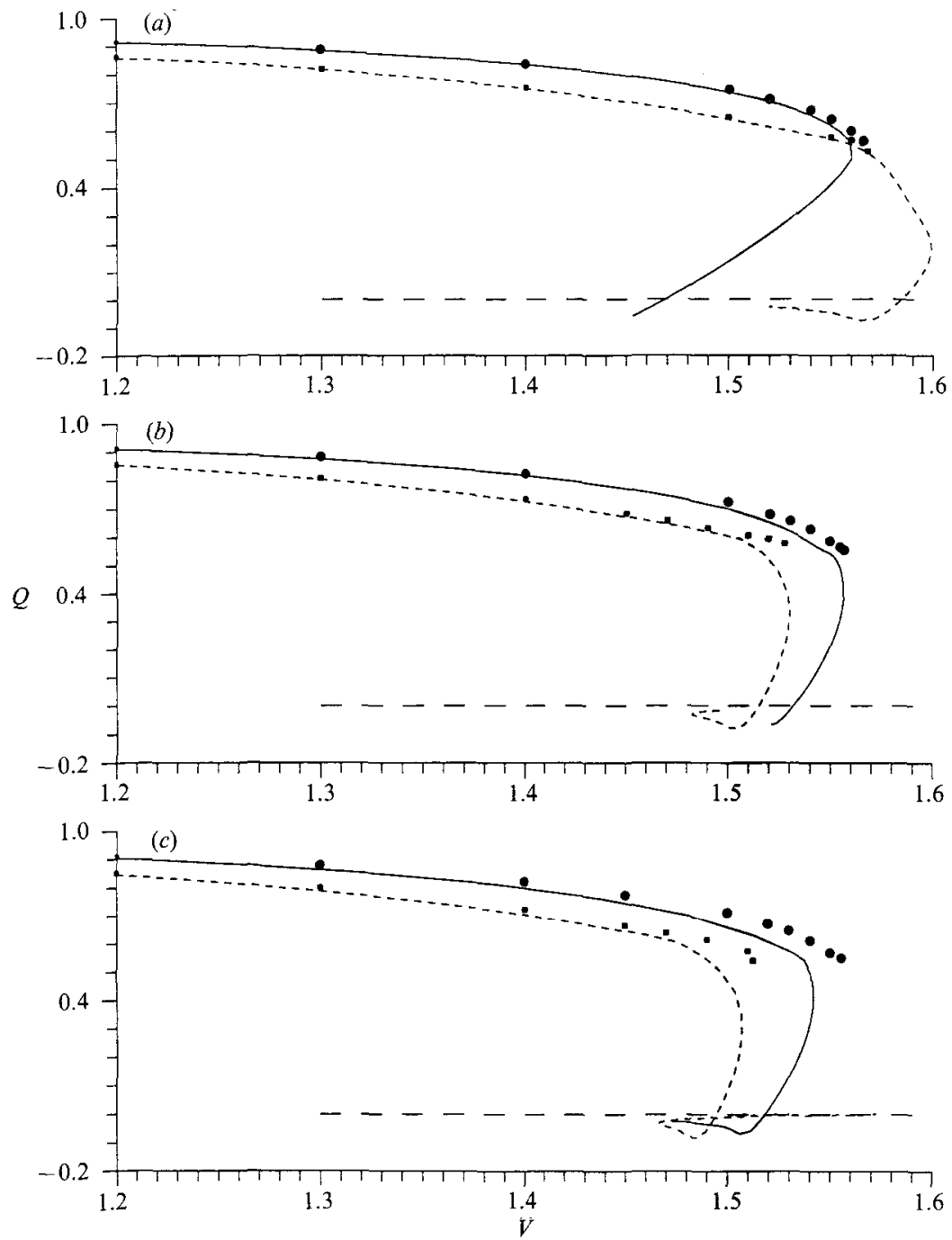

FigURE 7. Solution diagrams for the Navier-Stokes equations with,$--- R e=1000$ and - , $R e=2000$ and the $\mathrm{QC}$ equations with $\mathbf{\square}, R e=1000$ and $\operatorname{Re}=2000:(a) \alpha=0.0 ;(b) \alpha=0.025$; (c) $\alpha=0.05$.

takes place when $Q<0$. The plots serve to illustrate several points. First, for the three largest values of the Reynolds number examined, non-unique solutions of the Navier-Stokes equations were computed over varying ranges of the vortex circulation for all three values of $\alpha$. When $R e=250$, non-uniqueness was only observed for the straight pipe $(\alpha=0)$. Reversed flow was found in all cases when the vortex was specified to be sufficiently strong. However, all solutions corresponding to primary limit points were observed to be free of recirculation, indicating that the onset of reversed flow is neither a necessary nor sufficient condition for large changes in flow behaviour. As Reynolds number was increased, the value of $Q$ at the primary limit point was found to also increase.

With the relatively coarse grids that were used to calculate the paths SP1-SP12, convergence was a common problem once the flow had become reversed. Continuation could only be extended by taking very small values of the continuation 

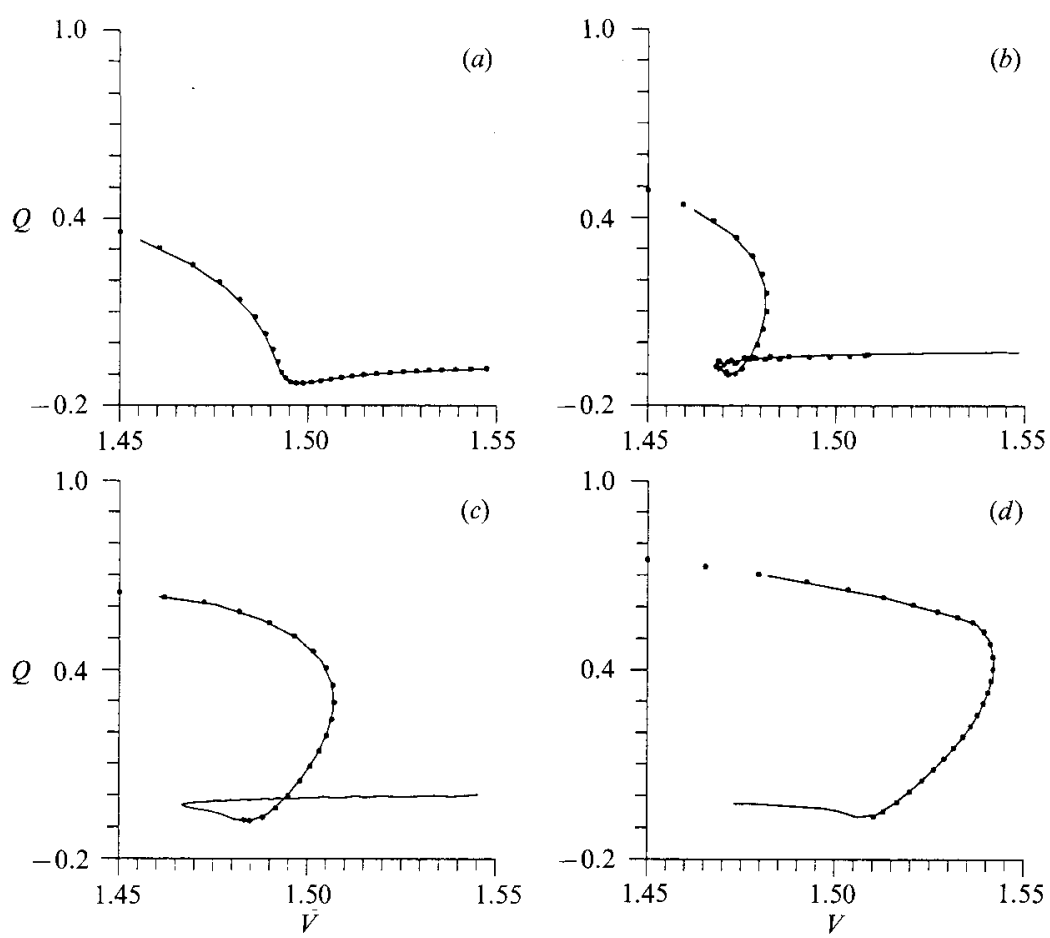

FIGURE 8. Solution sensitivity to axial node spacing, $\Delta_{2}$, using $\bullet, I=151$ and,$- I=301$ with $\alpha=0.05, J=27$, and $(a) R e=250(b) R e=500(c) R e=1000(d) R e=2000$.

parameter, $d$. In some cases, this did not seem worthwhile, because the solution paths showed signs of spurious behaviour through the development of numerous minor limit points, and because of the increased computational expense. Spurious behaviour was eliminated when the axial node spacing was reduced, as will be described below.

The point at which the QC equations first failed with increasing circulation was found to correlate reasonably well with the location of the primary limit point when the Reynolds number was 1000 or 2000 . At the lowest Reynolds number examined, 250 , there is least agreement, which may be attributable to the loss of accuracy of the QC equations at low Reynolds numbers where the boundary-layer approximation is weakest. In comparing the profiles of figures $7(b)$ and $7(c)$, there also appears to be some loss of correlation between the QC and Navier-Stokes equations with increased $\alpha$. This is again consistent with loss of accuracy of the QC equations as axial gradients become more pronounced. In this case, axial gradients are caused by the changing pipe geometry.

It is evident in both figures 6 and 7 that the effect of increasing $\alpha$ was the development of reversed flow at lower values of vortex strength. This observation is consistent with the finding of Sarpkaya (1974) that an adverse pressure gradient encourages the formation of vortex breakdown. In this study, an adverse pressure gradient is imposed on the flow downstream of the throat owing to the expansion of the pipe.

\subsection{Solution sensitivity to node spacing}

For a specified pipe geometry $(\alpha=0.05)$, the node spacing was varied to assess the sensitivity of solutions of the Navier-Stokes equations to $\Delta_{\xi}$ and $\Delta_{\xi}$. To determine the sensitivity to axial node spacing, solution paths were computed for $I=151$ and four 

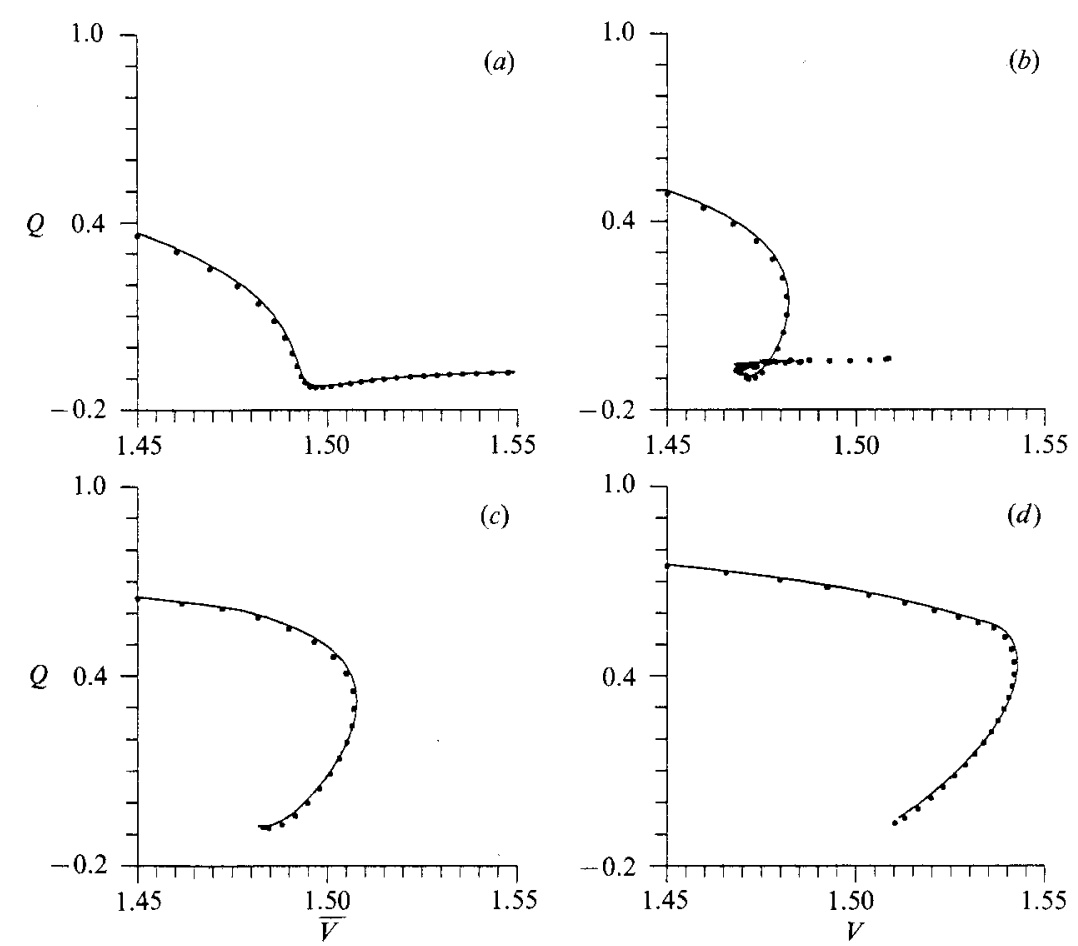

Figure 9. Solution sensitivity to radial node spacing, $\Delta_{r}$, using $\bigcirc, J=27$ and,$- J=40$ with $\alpha=0.05, I=105$, and $(a) R e=250(b) R e=500(c) R e=1000(d) R e=2000$.

different values of Reynolds number and then compared with the 'fine-grid' paths: SP1, SP4, SP7 and SP10. A similar comparison was made for the effects of decreased radial node spacing by computing solutions with $J=40$ and $I=151$. Finally, solutions were obtained with the most refined grid, G6, and compared with those computed on coarser grids.

Solution paths are compared in figures 8 and 9 . Figure 8 shows that the increase of $\Delta_{\xi}$ had little effect on the qualitative or quantitative behaviour of any of the coarse-grid paths, except in the vicinity of secondary limit points. With the fine grid, a distinct secondary limit point, free of spurious behaviour, is computed for $R e=500$. With coarse grids, convergence was generally difficult to achieve and calculations were halted prior to computation of secondary limit points. However, for $R e=500$, calculations were continued beyond a pronounced secondary limit point, after which the solution path was found to exhibit spurious behaviour. This behaviour was marked by the formation of a large number of successive limit points.

When $\Delta_{\zeta}$ was decreased by increasing $J$ with $I=151$, some small quantitative differences between the fine-grid and coarse-grid results were observed for all four values of Reynolds number. These differences were typically in a direction opposite to the smaller differences observed in figure 8 . In contrast to results obtained through variation of $\Delta_{\xi}$, the decrease of $\Delta_{\zeta}$ did not remove the spurious behaviour near secondary limit points.

To further confirm the ineffectiveness of decreased $\Delta_{\zeta}$ in reducing spurious behaviour, solutions paths were computed for $J=27$ and $J=40$ at four different Reynolds numbers with $I=301$. These paths are compared in figure 10 . At $R e=500$, the two solution paths are free of spurious behaviour; increased $J$ has little effect on 

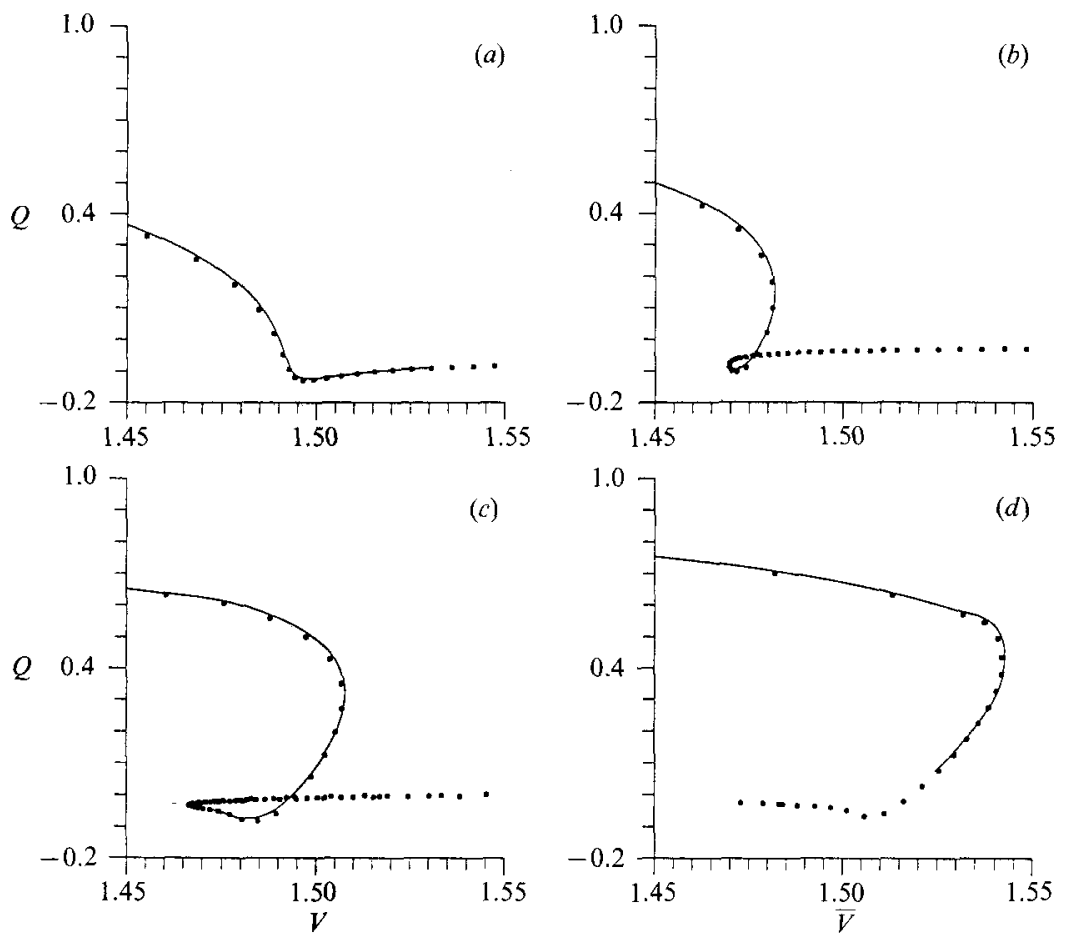

Figure 10. Solution sensitivity to radial node spacing, $\Delta_{r}$, using $\boldsymbol{O}, J=27$ and,$- J=40$ with $\alpha=0.05, I=301$, and $(a) R e=250(b) R e=500(c) R e=1000(d) R e=2000$.

$\begin{array}{ccccc}\text { Solution point } & \text { Solution path } & \text { Grid } & \text { Re } & V \\ \text { P1 } & \text { SP1 } & \text { G3 } & 250 & 1.45 \\ \text { P2 } & \text { SP1 } & \text { G3 } & 250 & 1.50 \\ \text { P3 } & \text { SP1 } & \text { G3 } & 250 & 1.55 \\ \text { P4 } & \text { SP7 } & \text { G3 } & 1000 & 1.48 \\ \text { P5 } & \text { SP7 } & \text { G3 } & 1000 & 1.48 \\ \text { P6 } & \text { SP7 } & \text { G3 } & 1000 & 1.48 \\ \text { P7 } & \text { SP9 } & \text { G5 } & 1000 & 1.53 \\ \text { P8 } & \text { SP9 } & \text { G5 } & 1000 & 1.53\end{array}$

TABLE 5. Selected solution points on three paths: SP1, SP7 and SP9

computed solution points. At the largest Reynolds number, Re $=2000$, increasing $J$ has a small effect on the location of the primary limit point, but does not improve convergence; with $J=40$ the continuation procedure fails at approximately $V=1.525$.

We anticipate that for $R e$ below approximately 2000 , accuracy of smooth solutions obtained with $I=301$ and $J=27$ would be minimally improved through grid refinement. Also, the large computational requirements imposed by the solution technique prevented the use of grids finer than that already described. However, we expect the accuracy and smoothness of spurious solutions to be improved by a reduction of $\Delta_{\xi}$. 

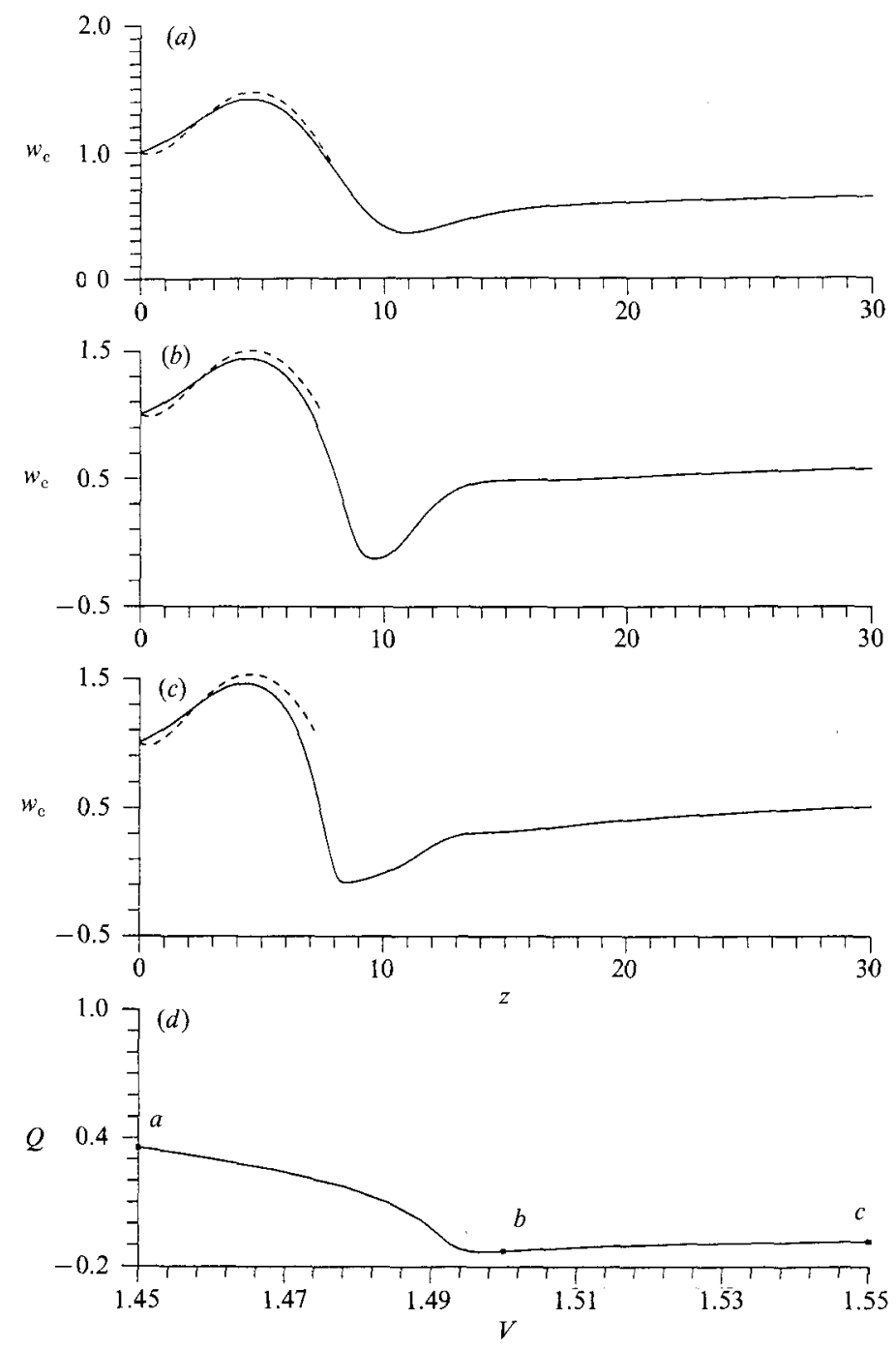

Figure 11. Profiles of centreline axial velocity for - - , the Navier-Stokes equations and ---, the $\mathrm{QC}$ equations with $R e=250$ at three different vortex strengths: $(a) V=1.45 ;(b) V=1.50 ;(c)$ $V=1.55 ;(d)$ three solution points located on the solution path.

\subsection{Solution points}

Eight points on three solutions paths of the Navier-Stokes equations, SP1 and SP7 $(\alpha=0.05)$, and SP9 $(\alpha=0.0)$, were examined in detail. The positions of these points and the paths they correspond to are summarized in table 5 . For $R e=1000, V=1.48$ and $\alpha=0.05$, three solutions were computed: before the primary limit point $(\mathrm{P} 4)$; before the secondary limit point (P5), and after the secondary limit point (P6). Solutions $\mathrm{P} 5$ and $\mathrm{P} 6$ have regions of recirculation. For $R e=1000, V=1.53$ and $\alpha=0.0$, two solutions were computed : before the primary limit point (P7), and after the primary limit point $(\mathrm{P} 8)$. The $\mathrm{Re}=250$, limit points are not observed and solutions are unique.

Figures 11 and 12 provide profiles of centreline axial velocity, $w_{c}$, and contour plots of streamfunction, respectively, for three points on the path $\operatorname{SP} 1(R e=250)$. 
(a)

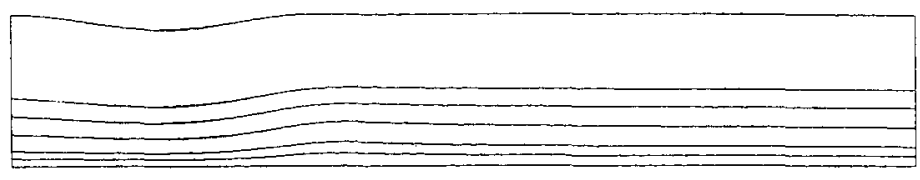

(b)

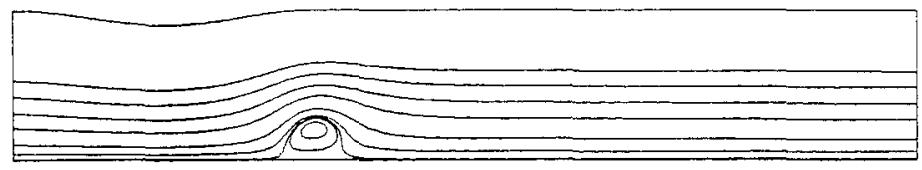

(c)

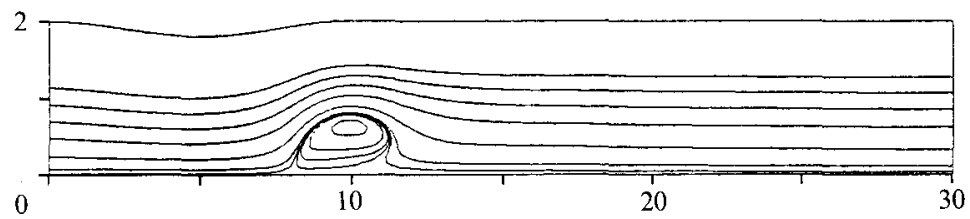

FigURE 12. Streamsurfaces corresponding to the three points selected in figure $11(R e=250)$.

Figure $11(d)$ shows a portion of the path on which the solution points are located. Figures $11(a)-11(c)$ also include profiles of $w_{\mathrm{c}}$ obtained through integration of the QC equations up to the point of failure (indicated by dashed lines).

The profiles in figures $11(a)$ and $11(b)$ are quite similar in character; the reversed flow at $V=1.50$ apparently evolves through a simple reduction in centreline axial velocity in the vicinity of the global velocity minimum without any other important change in behaviour. As $V$ is increased to 1.55 , the reversed flow region grows in size and takes on a shape that indicates a tendency towards flow entrainment near the aft portion of the breakdown bubble as observed by Grabowski (1974). The profiles in figures $11(a)-\mathbf{1 1}(c)$ are nearly identical in the vicinity of the inflow boundary. Also, when reversed flow develops the recirculation region is far from the same boundary, indicating that pipe constriction was successful in isolating this region from the inflow boundary. Furthermore, the $\mathrm{QC}$ equations provide a reasonable approximation to flow behaviour near the inflow boundary, indicating that in this region the flow remains quasi-cylindrical despite the low value of the Reynolds number.

The QC equations fail between one to two core radii upstream of the point at which the flow first becomes reversed. This result is consistent with the situation described by Leibovich (1989): 'The flow must have first become subcritical to axisymmetric disturbances before the station at which the stagnation point is located.' The critical point at which the flow changes from supercritical to subcritical is the station at which the QC equations become singular and numerical integration fails (Hall 1972; Trigub 1985). The QC equations also have the undesirable property of predicting breakdown when $V$ is sufficiently close to $V_{1 p}$, before recirculation is evident (e.g. at $V=1.45$ ). In these cases, flows have become subcritical at some stations without developing axial stagnation points.

Figures 13 and 14 provide profiles of $w_{\mathrm{c}}$ and contour plots of $\psi$, respectively, for three points on the path SP7 $(R e=1000)$. Figure $13(d)$ shows a portion of the path on which the solution points are located. This path has a very different character to that of SP1, for which no limit points were computed. For SP7, three equilibrium 

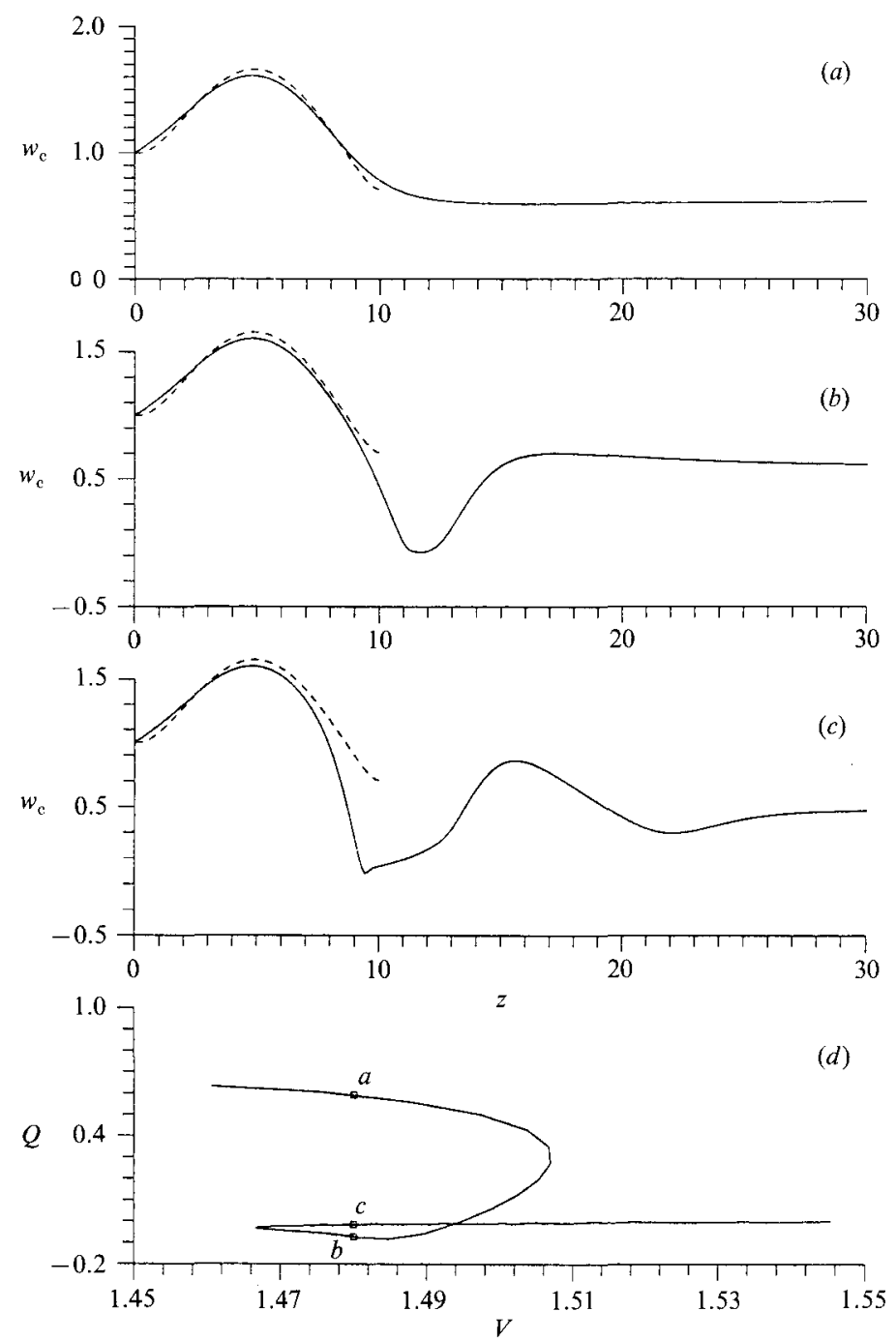

Figure 13. Profiles of centreline axial velocity for - - the Navier-Stokes equations and ---, the QC equations with $R e=1000$ and $V=1.48$ at three different solutions points: $(a)$ upper branch ; $(b)$ lower branch; $(c)$ intermediate branch; $(d)$ three solution points located on the solution path.

solutions are available for vortex strengths ranging between 1.4666 and 1.5069 . Figures $13(a)-13(c)$ also include profiles of $w_{\mathrm{c}}$ obtained through integration of the QC equations (indicated by dashed lines).

As was found true for $R e=250$, there is little qualitative difference between the profiles of $w_{\mathrm{c}}$ corresponding to solutions P4 and P5, even though solution P5 contains reversed flow. Although smaller, the bubble seen in figure $14(b)$ is also quite similar to that shown in figure $12(b)$. As arclength is increased along the path from solution P5 to P6, the reversed flow region grows dramatically and becomes nearly disconnected from the centreline of the pipe, causing $w_{\mathrm{c}}$ to be negative for only a small axial distance. The recirculation region also moves upstream with increased circulation, in agreement with other physical and numerical experiments (e.g. Lambourne \& Bryer 1961; Grabowski 1974). However, the breakdown region does not move far enough upstream to have a noticeable effect on $w_{\mathrm{c}}$ near the inflow 
(a)

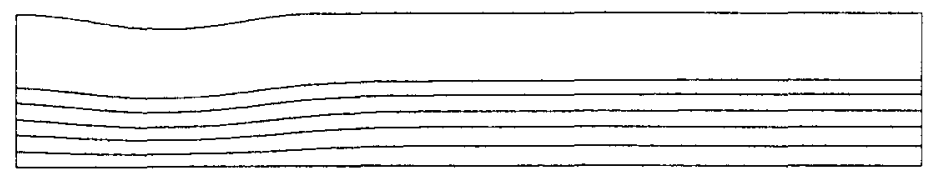

(b)

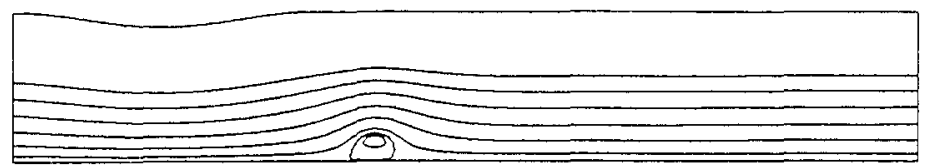

(c)

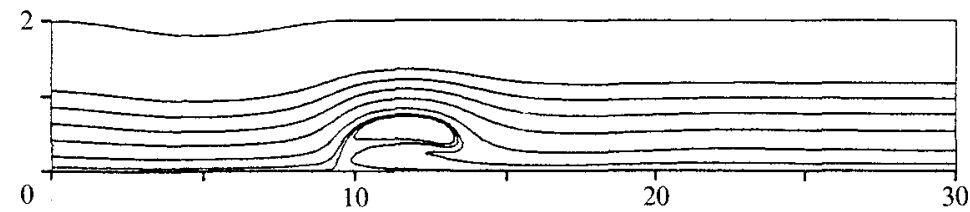

FrguRE 14. Streamsurfaces corresponding to the three points selected in figure $13(R e=1000)$.

boundary where core fluid elements are accelerated. This point is illustrated in figure $15(a)$ where $w_{\mathrm{c}}$ is compared for all three solution points. Again, the QC equations provide a reasonable approximation to flow behaviour near the inflow boundary, although as the core flow decelerates, the QC equations ultimately fail.

Diagnostic parameters other than $Q$ may be used to present computed paths in solution diagrams. As an example, the minimum value of the azimuthal component of vorticity.

$$
Q_{\eta} \equiv \min _{k}\left[\eta\left(\xi_{k}, \zeta_{k}\right)\right]
$$

is plotted against $V$ in figure $15(b)$ for paths SP7 and SP9. Use of the diagnostic $Q_{\eta}$ yields a path shape close to the one depicted in figure 1 and somewhat different to the one shown in figure $13(d)$.

Figure $15(b)$ also serves to illustrate the effects of $\alpha$ variation on path shape and solution behaviour. When the pipe is straight $(\alpha=0)$, the solution path SP9 maintains the characteristic reversed ' $S$ '-shape of SP7. However, as illustrated in figure $15(c)$, the solutions $\mathrm{P} 7$ and P8 (their relative positions are shown in figure $16 b$ ) do not have the property that the centreline axial velocity near the inflow boundary is independent of path position at constant $V$. The primary reason for the examination of pipes with varying radius $(\alpha>0)$ lies in the removal, in the minds of the authors, of the inflow conditions as potential causes for the existence of multiple flow states. For example, it is unclear from the velocity profiles plotted in figure $\mathbf{1 5}(\mathrm{c})$ (or others obtainable at different Reynolds numbers and circulations) whether the close proximity of the reversed flow region to the inflow boundary influences the behaviour of the emerging breakdown structure. With $\alpha=0.05$, a sizeable region of supercritical flow exists between the breakdown structure and the inflow boundary, so that the presence of the reversed flow region is not reflected in the conditions specified at the inlet.

As observed in several numerical studies of vortex breakdown, including Krause et al. (1983), Brown \& Lopez (1988) and Salas \& Kuruvila (1989), breakdown in a straight pipe inevitable occurs near the inlet. In a time-dependent sense, once a 

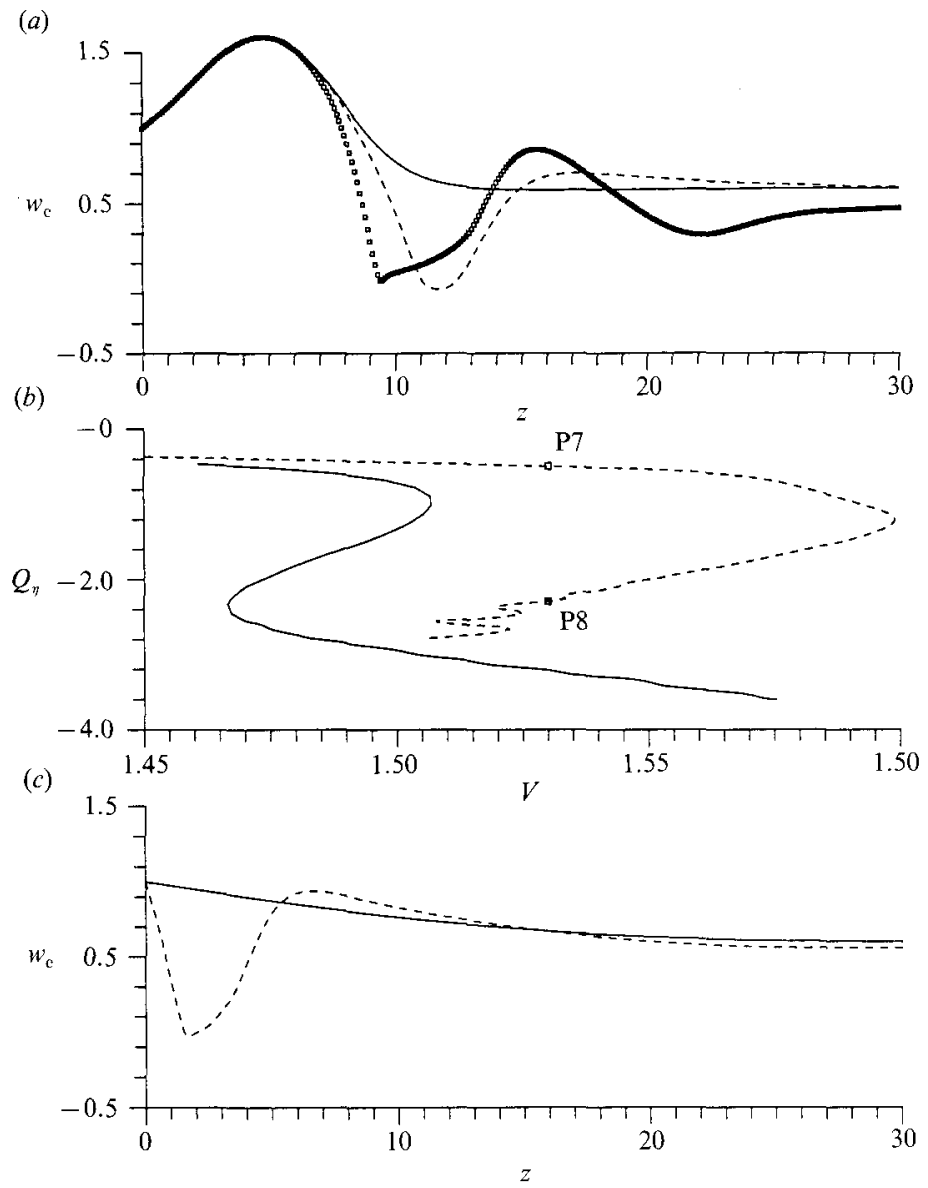

Figure 15. (a) Profiles of centreline axial velocity for the three solution points examined in figure 13: upper branch; ---, lower branch; $\square$, intermediate branch. (b) Two solution paths for $R e=1000:-, \alpha=0.05 ;---, \alpha=0.0$. (c) Profiles of centreline axial velocity for the two solution points examined in figure $15(b):-$, P7; ---, P8.

stagnation point forms, the breakdown structure grows and moves upstream until reaching the inlet. Consequently, axial gradients in this region are large. When the pipe is constricted $(\alpha>0)$, the breakdown structure is prevented from moving past the throat. The plots of streamsurface in figures 12 and 14 indicate that breakdown occurs at or just upstream of the end of the pipe's first stage $(z \approx 10)$. Although not reported in detail herein, the authors investigated flows in pipes with first stages five and six times longer than that of the standard domain. This was done to better model the long divergence sections of tubes used in vortex breakdown experiments (e.g. Faler \& Leibovich 1977). It was found that breakdown also occurred just upstream of $z=L$ for these pipes.

\subsection{Behaviour at low Reynolds number}

A bifurcation diagram showing calculated positions of primary and secondary limit points in the $R e-V$ parameter space (assuming $\alpha=0.05$ ) is provided in figure 16 for low Reynolds numbers (Re $\leqslant 1000)$. It is seen that the two branches of limit points converge to nearly a cusp at a critical Reynolds number, $R e_{\mathrm{c}}$, of approximately 360 . When $R e<R e_{\mathrm{c}}$, only unique solutions are computed. 


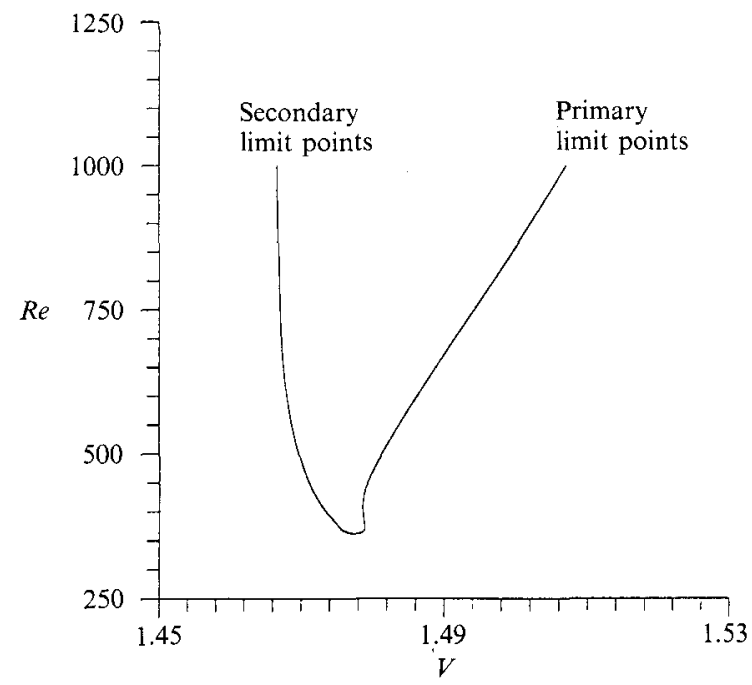

Figure 16. Bifurcation diagram showing location of primary and secondary limit points as functions of Reynolds number and vortex strength with $\alpha=0.05$.
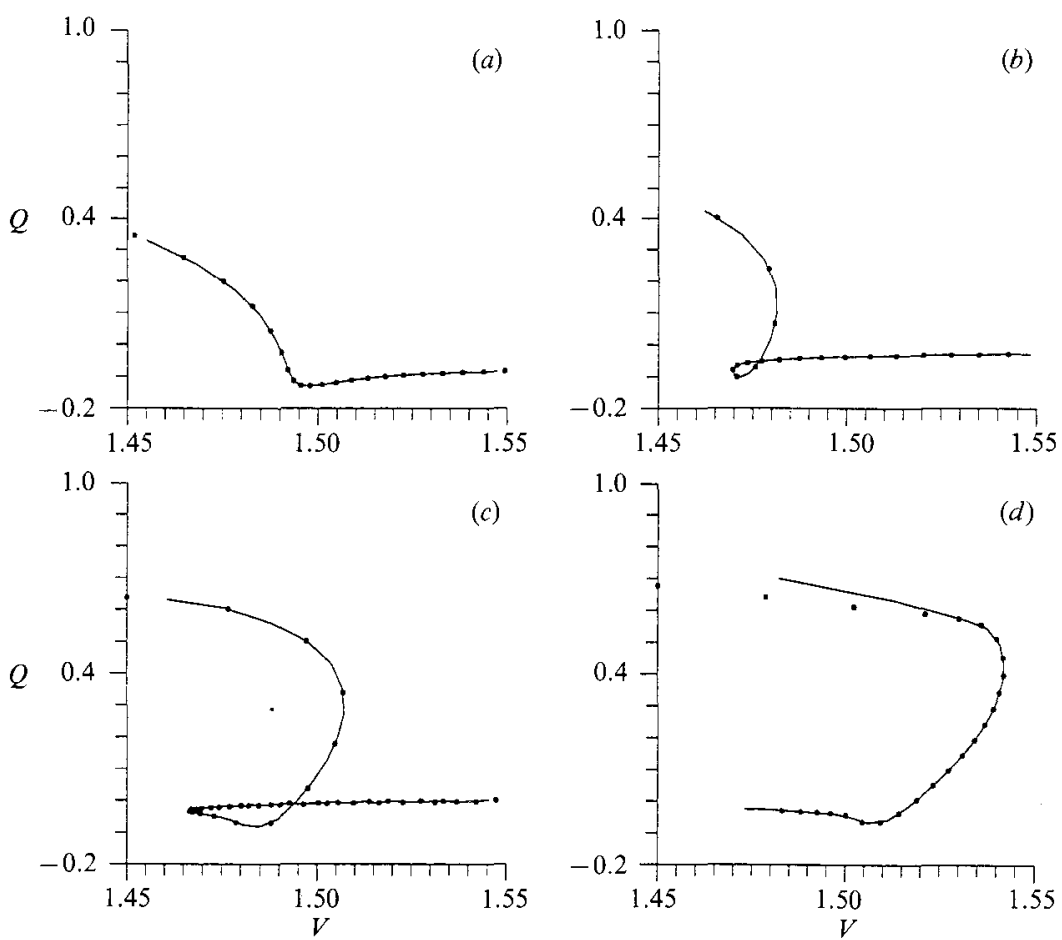

FigUre 17. Solution sensitivity to pipe length, $Z$, using $\bullet, Z=30$ and,$- Z=60$ with $\alpha=0.05$ and $(a) R e=250(b) R e=500(c) R e=1000(d) R e=2000$.

\subsection{Solution sensitivity to pipe length}

A study of solution sensitivity to domain length, $Z$, with $L$ fixed for relatively low Reynolds numbers (Re $\leqslant 2000$ ) was performed. Solution sensitivity to $Z$ was not investigated for larger Reynolds numbers owing to the greatly increased com- 
putational expense associated with performing calculations over very long physical domains. Solution paths were computed for $\alpha=0.05$ with a lengthened pipe, $Z=6 L=60$ (grid G8), at four Reynolds numbers: 250 (SP25), 500 (SP26), 1000 (SP27) and 2000 (SP28). Figure 17 shows the solution diagrams for all four cases; solution paths are compared to those obtained with a pipe of length $3 L$ (grid G3). The comparison indicates that for Reynolds numbers not exceeding 1000, vortex behaviour can be effectively modelled with the shorter pipe. When $R e=2000$, there are quantitative differences in $Q$ that arise from the inability of the shorter pipe to capture the full extent of vortex decay. At smaller values of $Q$, comparison is better, since then the axial flow is most decelerated far upstream of the outflow boundary.

\subsection{Solution sensitivity to pipe radius}

Solution sensitivity to domain radius, $R_{0}$, was examined for relatively low Reynolds numbers (Re $\leqslant 2000$ ) by comparing solutions for $R_{0}=3$ with those computed assuming $R_{0}=2$. Solution paths were computed for $\alpha=0.05$ and $R_{0}=3$ with grid G7 at four Reynolds numbers: 250 (SP29), 500 (SP30), 1000 (SP31) and (SP32). These paths are compared in figure 18 to paths SP1, SP4, SP7 and SP10. Comparison indicates that for the range of Reynolds numbers and pipe radii examined, vortex behaviour is insensitive to pipe radius. Pipes wider than $R_{0}=3$ were prohibitively expensive to examine, assuming $\Delta_{\zeta}=\frac{1}{13}$.

\subsection{Comparison with results of the Euler equations}

The Euler equations governing the axisymmtric, swirling flow of an inviscid fluid may be simplified to a single, nonlinear equation for the streamfunction, $\psi$. This equation, attributed by Leibovich \& Kribus (1990) to Bragg \& Hawthorne (1950), will be referred to as the BHE and is given by

$$
\psi_{z z}+\psi_{r r}-\frac{1}{r} \psi_{r}=r^{2} \dot{H}-\Gamma \dot{\Gamma}
$$

The circulation, $\Gamma$ (defined in $(5 a)$ ), is solely a function of $\psi$ in inviscid flow. The total head, $H$, is also a function of $\psi$ only:

$$
H(\psi)=\frac{p}{\rho}+\frac{1}{2}\left(u^{2}+v^{2}+w^{2}\right)
$$

The explicit dependence of the right-hand side of $(51)$ on $\psi$ is obtained through the specification of the flow state at a particular axial station. The flow field, of which the flow state is a component, is termed the 'specifying flow' by Leibovich \& Kribus and is typically chosen to be columnar. Through construction, the specifying flow identically satisfies the BHE.

Non-unique solutions of the BHE have been previously computed for swirling flows in straight pipes by Táasan (1986) and Leibovich \& Kribus (1990). Figure 19 is a characterization of the composite solution space revealed by both investigations. Táasan identified a solution branch (branch III in figure 19) of soliton solutions of the BHE, which bifurcates from the path of specifying flow solutions (branch I in figure 19). A bifurcation point (point $A$ in figure 19) marks the critical state, separating 'supercritical' flow states (columnar solutions at vortex strengths below the critical value from 'subcritical' flow states (columnar solutions at vortex strengths above the critical value). Soliton solutions experience flow deceleration in the vortex core and ultimately achieve flow reversal when the vortex strength becomes sufficiently 

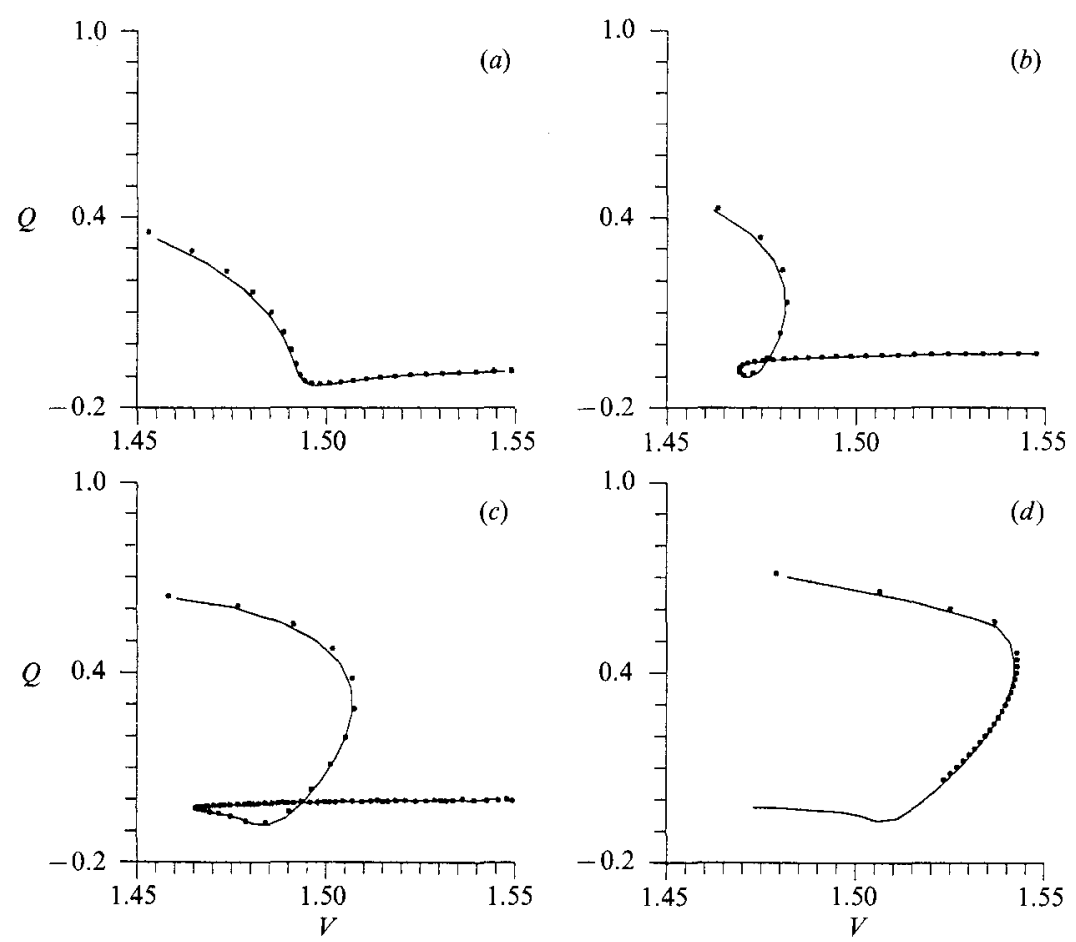

FIGURE 18. Solution sensitivity to pipe radius, $R_{0}$, using $\bullet, R_{0}=3$ and,$- R_{0}=2$ with $\alpha=0.05$ and $(a) R e=250(b) R e=500(c) R e=1000 \quad(d) R e=2000$.

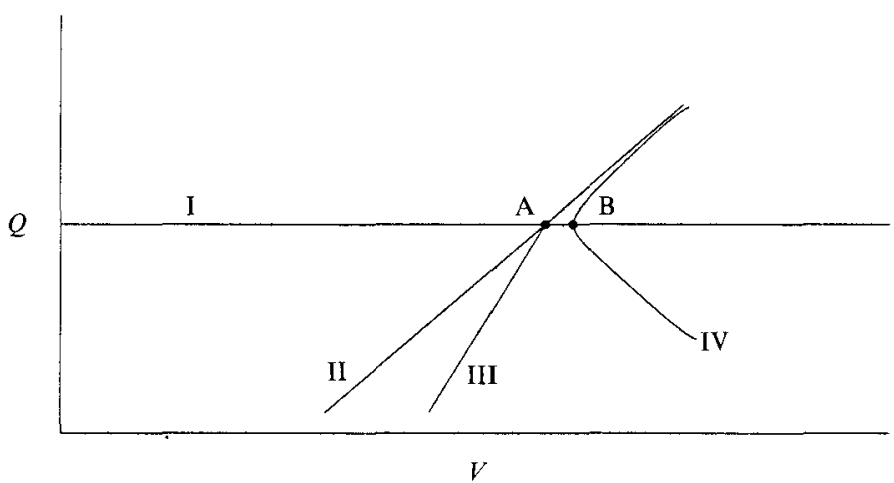

FIGURE 19. Characteristic solution diagram for the Bragg-Hawthorne equation, including four selection branches: (I) trivial branch; (II) principal conjugate branch; (III) soliton wave branch; (IV) wavetrain branch, and two bifurcation points; (A) primary; (B) secondary.

small. Leibovich \& Kribus explored the solution space for a similar class of specifying flows and discovered two additional solution branches: a branch of columnar solutions (branch II in figure 19) representing the principal conjugate flows (in the sense defined by Benjamin (1962) and Benjamin (1967)), and a branch composed of wavetrain solutions (branch IV in figure 19). Branches $I$ and IV intersect at a secondary bifurcation point (point $B$ in figure 19) where the flow is subcritical.

Solution paths of the Navier-Stokes equations are found in this investigation to be distinctly different to those of the Euler equations owing to the presence of limit points and the absence of bifurcation points. Flow behaviour at high Reynolds 


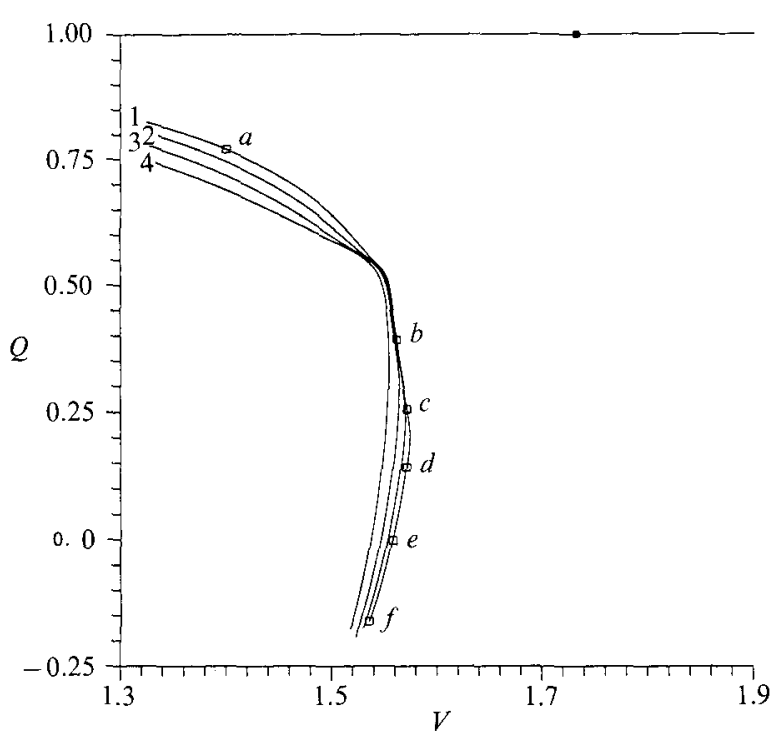

FIgURE 20. Solution diagram including four solution paths: (1) SP33 (Re $=6000)$ (2) SP34 $(R e=5000)(3)$ SP35 $(R e=4000)$ and (4) SP36 $(R e=3000)$; six solution points on path $(1):(\alpha)$ $V=1.4(b) V=1.5617(c) V=1.5714(d) V=1.5708(e) V=1.5576$ and $(f) V=1.5348$. 9 , primary bifurcation point on the trivial path of the BHE located at $V=1.7325$.

number is examined with four solution paths $(\alpha=0.05)$ : SP33 $(R e=6000)$, SP34 $(R e=5000)$, SP35 $(R e=4000)$, and SP36 $(R e=3000)$. At such large Reynolds numbers, the authors found it necessary to use grid G9, which has a second stage long enough to capture most significant flow features. However, to maintain a sufficiently small axial node spacing while limiting the total number of nodes to a manageable number, it was also found necessary to decrease the number of nodes in the radial direction to 14 . The effects of this reduction were not examined, although, as seen in figure 10 , solutions show little dependence on $J$ beyond $J=27$. The effects of varying $\alpha$ over the same ranges of $R e$ and $V$ were also not investigated.

The paths SP33-SP36 are presented in figure 20 along with 'trivial', but noncolumnar solutions of the BHE for $\alpha=0.05$ (points lying on the path defined by the family of specifying flows). These solutions were computed on grid G9 in a manner similar to that described in $\$ 3$. The boundary conditions enforced on $\psi$ are equivalent to those used in the analysis of the viscous flow equations. The specified profiles of $\Gamma$ and $\eta$ at the inflow boundary $((21 b)$ and $(23))$ were used to determine the form of the right-hand side of (51). The purpose of examining the BHE in this investigation was to compute the location of the primary bifurcation point on the trivial path (corresponding to point A in figure 19). Calculation of this location was achieved by examining the sign of the determinant of the Jacobian computed at each Newton iterate as vortex strength was systematically varied. For the family of specifying flows studied here, the location of the primary bifurcation point was found to be $V=1.7325$ (as plotted in figure 20 ).

Each of the four solution paths of the Navier-Stokes equations shown in figure 20 display markedly different behaviours in three distinct path segments. At small vortex strengths $(Q \approx 1)$ the centreline axial velocity, $w_{c}$, is found to decrease monotonically from the peak value (achieved at the pipe throat) with increasing $z$. When $V$ increases beyond $V_{\text {tr }}$, where $V_{\text {tr }}$ is approximately 1.55 , a wavetrain forms on 


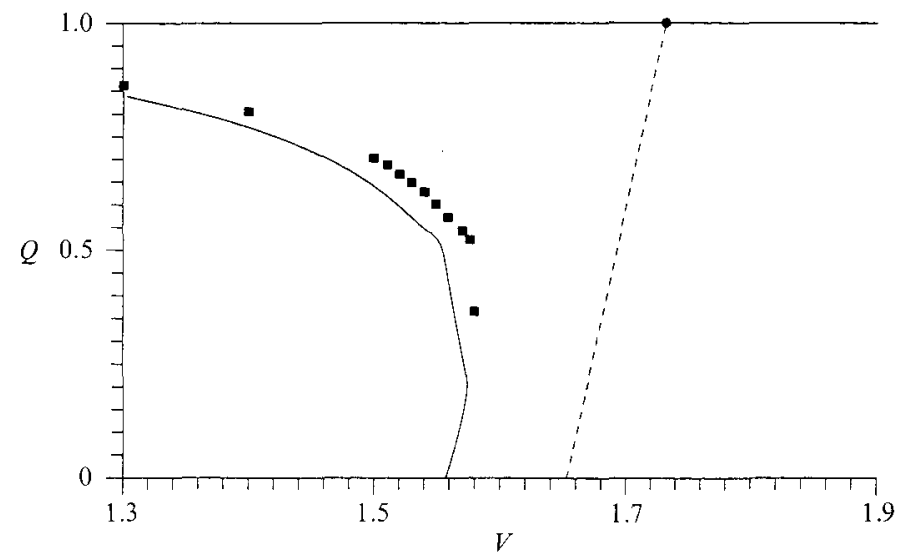

Figure 21. Solution diagram for ——, the Navier-Stokes equations and $\square$, the QC equations with $R e=6000$. - - , postulated branch approximately tangent to the path segment below the primary limit point $(Q<0.2055)$ intersects the bifurcation point of the BHE at $V=1.7325$.

the vortex. The wavetrain becomes more prominent with further increases in $V$. Development of wavetrain behaviour is accompanied by a rather abrupt change in the slope of the paths shown in figure 20 . At a critical value of the vortex strength, $V_{1 p}$, a limit point is encountered, beyond which the solution path turns back on itself. The branch 'below' the limit point is composed of solutions that are intermediate states between flow states that exhibit solitary and wavetrain behaviour.

At Reynolds numbers below about 1000 (assuming $\alpha=0.05$ ), solution paths computed through the variation of $V$ do no display a marked change in slope before the primary limit point. Wavetrain solutions are also not observed. Solution paths were not computed for Reynolds numbers exceeding 6000 , since at such large values the continuation process would often fail.

The values of $V_{t r}$ at which the solution paths began to develop a wavetrain behaviour are nearly equivalent to $V_{\mathrm{lp}}$ and the values of $V$ at which the QC equations first become singular, $V_{c}$. This observation is illustrated in figure 21 , which shows the solution path SP33 (Re $=6000)$ plotted against solution points obtained through integration of the QC equations (using grid G13). In comparison with the results of the Euler equations characterized in figure 19, solutions of the Navier-Stokes equations exhibit a behaviour that would be expected from the movement of the secondary bifurcation point (point $B$ ) to a supercritical location before the primary bifurcation point $\left(V_{\mathrm{B}}<V_{\mathrm{A}}\right)$. In this scenario, SP33 is attracted to the branch of wavetrain solutions (IV), preferentially choosing the lower portion of branch IV owing to the viscous decay of the vortex core.

Failure of the QC equations at a value of $V$ near the circulation at which wavetrain behaviour first develops is consistent with the contrasting wave propagation characteristics of supercritical and subcritical flows. Flows that are entirely supercritical cannot support standing waves (Benjamin 1962), and can be calculated through integration of the $\mathrm{QC}$ equations. Flows that become subcritical over a portion of their extent support standing waves, and must possess a critical state where the flow changes from supercritical to subcritical. Thus, failure of the QC equations and wavetrain development are manifestations of the same event: the formation of subcritical flow. At the critical state, the QC equations are singular through an inviscid mechanism (Hall 1972; Trigub 1985). The inviscid nature of the 

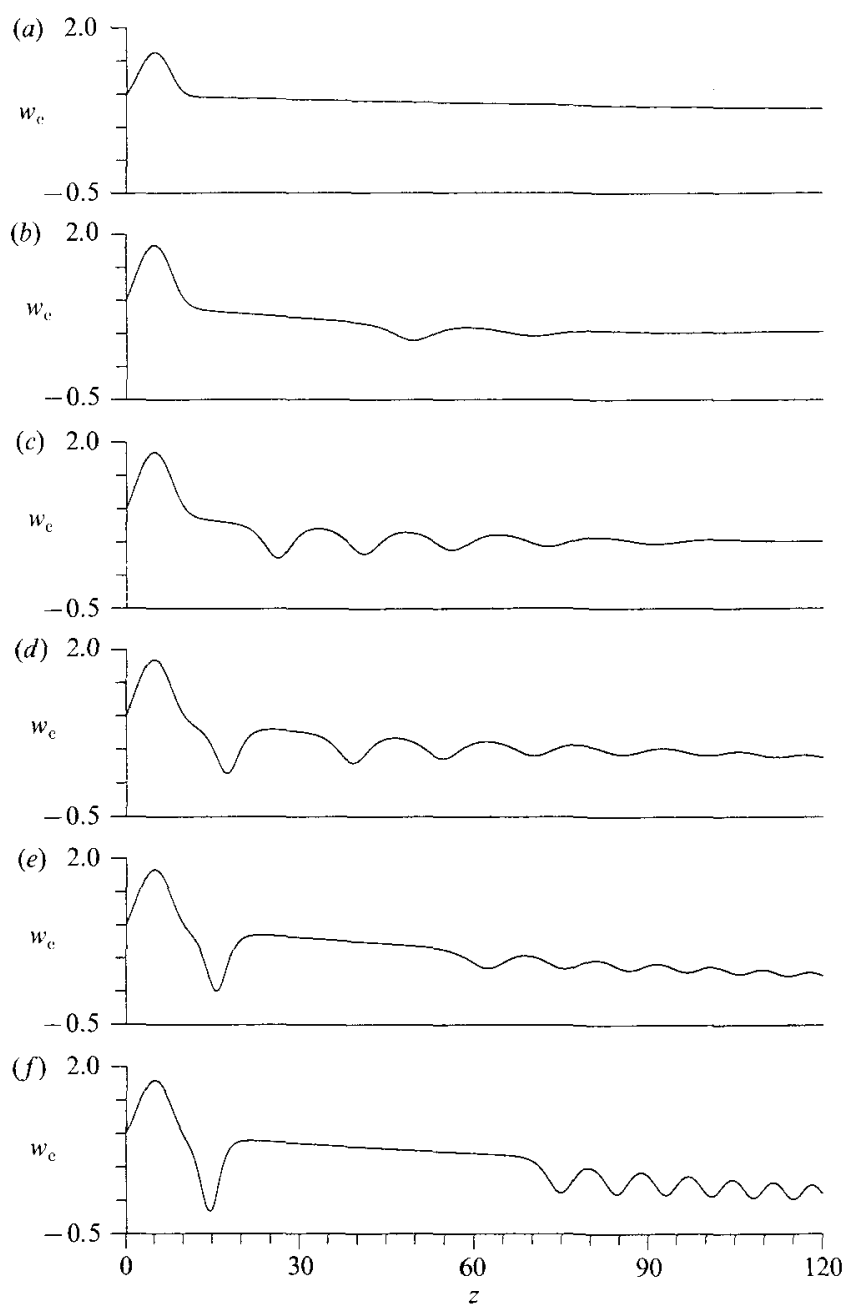

Figture 22. Profiles of centreline axial velocity for the six solution points introduced in figure 20 :

(a) $V=1.4(b) V=1.5617$ (c) $V=1.5714$ (d) $V=1.5708$ (c) $V=1.5576$ and $(f)=1.5348$.

singularity is evident here through the relative insensitivity of $V_{\mathrm{c}}$ to changes in $R e$. The results above suggest that the inviscid mechanism is a singularity present in the Euler equations, the secondary bifurcation point, B.

Wavetrain behaviour in solutions of the Navier-Stokes equations at high Reynolds numbers has been reported by Beran (1989) for trailing vortex flows. In that study, the wavetrain developed downstream of a region in which the vortex slowly decayed; the point at which the vortex changes behaviour was termed the 'transition' point. Beran found that following the appearance of a wavetrain in the solution, further increases of $V$ served to increase wavetrain length and the amplitude of wavetrain oscillations, and caused the transition point to occur closer to the inflow boundary.

These observations are consistent with the findings of this study, as illustrated in figure 22, which provides profiles of centreline axial velocity for the six solution points $(a-f)$ on the path SP33 (Re $=6000)$ marked in figure 20. As $V$ is increased from 1.5617 (point $b$ ) to 1.5714 (point $c$ ), the wavetrain grows from two to five minima in extent, the oscillations grow in amplitude, and the wavetrain moves upstream. 
Solutions $b$ and $c$ do not involve reversed flow, yet the effects of changes in $V$ on the position of the transition point in these two solutions is qualitatively the same as the effect of circulation on the breakdown position of tube vortices. As $V$ is increased, a breakdown bubble in the tube moves upstream, while a decrease in $V$ leads to the opposite result (Faler \& Leibovich 1977). The shapes of wavetrain components computed in this study are also in qualitative agreement with those described by Leibovich \& Kribus (1990): wave troughs (as reflected in the $w_{\mathrm{c}}$ profiles) are relatively sharp, while wave crests are 'broad and flat'.

Continuation in $V$ beyond point $c$ on path SP33 leads ultimately to the calculation of a limit point at $V=1.5739$. Solutions $d$, $e$ and $f$ below the limit were selected to represent the third path segment. In figure 22 , it is seen that with decrease of $V$, the wavetrain splits apart, leaving a solitary wave near the end of the first pipe stage. The bulk of the wavetrain moves downstream with decreasing $V$. It is evident that for points $e$ and $f$, grid G9 does not adequately capture the full extent of the retreating wavetrain; a lengthened pipe is required to determine the sensitivity of wavetrain behaviour to change in $Z$.

On the path segment below the limit point, solutions possess an emerging behaviour similar to solutions on branch III, the branch of soliton solutions computed by Leibovich \& Kribus (1990). With decreasing $V$, the wave near the inflow boundary becomes more isolated and is strengthened, as manifested through a continued drop in $Q$. A reasonable explanation for this trend is that branch III (placed hypothetically in figure 21 as a dashed line) serves as an attractor for solutions of the Navier-Stokes equations, and that the attraction is stronger as the viscous flow solutions 'move away' from the branch of inviscid, wavetrain solutions, branch IV.

At large Reynolds numbers (3000-6000), the authors were unable to compute secondary limit points; the continuation method would fail before these points were reached. Thus, solutions for $V>V_{1 p}$ were inaccessible. (We also found that continuation in $R e$, starting at a very small Reynolds number with $V>V_{\text {lp }}$, would fail at a Reynolds number below 3000.)

\section{Conclusions}

The numerical simulation of swirling flows in pipes indicates that when the Reynolds number is sufficiently large, vortex breakdown occurs abruptly with increased circulation as a consequence of the existence of non-unique solutions. For a given pipe geometry, multiple solutions of the Navier-Stokes equations arise through the development of folds in a three-parameter space defined by the free parameters vortex circulation, $V$, and Reynolds number, $R e$, and a scalar that represents the observed flow state. In this study, the minimum value of the centreline axial velocity, $Q$, served as a convenient scalar parameter to monitor.

When the Reynolds number exceeds a critical value, $R e_{\mathrm{c}}\left(R e_{\mathrm{c}} \approx 360\right.$ when the contraction parameter, $\alpha$, is 0.05 ), the impact of solution non-uniqueness becomes evident after a limit point is encountered on a solution path computed through variation of $V$. The authors do not propose that the existence of the limit point causes vortex breakdown; this phenomenon appears to be an inevitable consequence of increased vortex circulation. However, the results predict an abrupt change in vortex behaviour, from a state with no reversed flow to a state with at least one large region of recirculation, as $V$ is increased beyond the circulation at the primary limit point $V_{1 \mathrm{p}}$. 
At large Reynolds numbers, computed solution paths are composed of points characterized by three flow types: quasi-cylindrical, soliton and wavetrain. The quasi-cylindrical and wavetrain solutions exist at larger values of the minimum centreline velocity, $Q$, and when $V<V_{\text {lp }}$. These flow states do not exhibit reversed flow; however, $Q$ decreases with increasing $V$ for these solutions, eventually achieving a value of $Q_{1 \mathrm{p}}$ at the limit point. Solutions primarily of a solitary nature also exist for $V<V_{1 p}$, but on a branch of non-unique solutions for which $Q$ continues to decrease: $Q<Q_{1 \mathrm{p}}$. Solutions at large $R e$ and at circulations exceeding $V_{1 \mathrm{p}}$ could not be computed owing to failure of the continuation method. At smaller Reynolds numbers, wavetrain solutions are not readily apparent (their emergence with increasing $R e$ is evident in figure $15 a$ for $R e=1000$ ), while solitary-wave solutions are calculable for $V>V_{\mathrm{lp}}$. When $R e<R e_{\mathrm{c}}$, viscous forces are apparently strong enough to damp out wavetrain and limit-point behaviour; only unique solutions are computed.

Previous investigations, including that of Salas \& Kuruvila (1989) and Beran (1989), have revealed wavetrain solutions with multiple, large regions of reversed flow, apparently free of dispersion errors. Such solutions were not observed in this study; the authors can only speculate that the inability to compute them is due to insufficient grid refinement or the boundary conditions applied.

The computation of three classes of viscous flow is consistent with the three types of inviscid solutions of the BHE computed by Leibovich \& Kribus (1990): columnar, soliton and wavetrain. The results of this study suggest that the characteristic shape of the solution paths computed at large Re arises through the attraction of viscous flow solutions to various points in the space of inviscid solutions. The authors intend to study the evolution of a path, as Re is increased, towards a particular arrangement of inviscid solution branches by computing the solution space of the BHE for pipes with varying radius $(\alpha>0)$.

Limit-point behaviour is observed when the pipe is either straight $(\alpha=0)$ or constricted $(\alpha>0)$ - a dependence on the qualitative characteristics of the solution space on the constriction parameter, $\alpha$, was not observed. However, by using a pipe with a convergent-divergent section, the breakdown structure was prevented from moving to a point close to the inflow boundary and causing large axial gradients in flow quantities at that location. With small axial gradients near the inflow boundary, the solution remained consistent with the assumption of quasi-cylindrical flow implied by the enforcement of the Dirichlet condition on vorticity at that boundary. Thus, the development of limit points and non-unique solutions at sufficiently large values of $R e$ and $V$ is not an artifact of a non-physical boundary condition.

As $V$ is increased from a small value with Reynolds number fixed $\left(>R e_{\mathrm{c}}\right.$ ), the development of a primary limit point on the solution path of solutions to the Navier-Stokes equations is well predicted by the failure of the integration of the QC equations. In other words, the first appearance of the critical point of Hall's boundary-layer separation theory, the point of failure of the QC equations, is a good approximation of the limit point in the Navier-Stokes equations. Thus, the QC equations serve as an efficient means to determine at what point, in a parameter space, that breakdown will first occur. Furthermore, upstream of breakdown, where the flow is generally supercritical, the $\mathrm{QC}$ equations provide a reasonable approximation of the flow behaviour.

There are several limitations of this work that require further investigation. First, and most important, the role of three-dimensionality has not been assessed. Threedimensional effects would most probably be significant to the flow state in the 
vicinity of limit points and when reversed flow develops. Also, stability of the rotationally symmetric solutions was not determined, so that the applicability of this analysis is uncertain. Finally, a physical mechanism responsible for the development of limit points was not identified.

We gratefully acknowledge the support of the Air Force Office of Scientific Research through grant 2301-D2 and the supercomputer resources provided by the Ohio Supercomputer Center through Grant PIS003-1. We would also like to thank the editor and referees for their helpful contributions, especially in the formulation of the pipe wall boundary conditions on vorticity.

\section{Appendix}

As the QC equations are parabolic, the axial gradients of flow quantities at an axial station may be computed using known flow quantities. Expressions for $\psi_{z}(r)$ and $\Gamma_{z}(r)$, in terms of functions determinable at specified axial stations, are developed below. Expressions for $\psi_{\xi}(\zeta)$ and $\Gamma_{\zeta}(\xi)$ are also provided. These results are incorporated into a first-order, explicit algorithm used to integrate the QC equations (Beran 1989).

With the quasi-cylindrical assumption, the streamfunction equation can be expressed as

$$
\eta=-\frac{1}{r}\left(\psi_{r r}-\frac{\psi_{r}}{r}\right)
$$

Equation (A 1) indicates that $\eta$ is solely dependent on the local state of the streamfunction at a particular station. Thus, integration of the QC equations is ultimately based on two timelike equations, which are used to determine the axial gradients of streamfunction and circulation from the local flow state. Vorticity serves only as an intermediate coupling variable, much like pressure in the time-dependent, incompressible flow equations. Differentiation of (A 1 ) with respect to axial position yields

$$
\eta_{z}=-\frac{1}{r}\left(\psi_{z r r}-\frac{\psi_{z r}}{r}\right)
$$

With the definition of streamfunction, $u \equiv-\psi_{z} / r$, (A 2) becomes

$$
\eta_{z}=u_{r r}+\frac{u_{r}}{r}-\frac{u}{r^{2}}
$$

Equation (7) can be solved for the axial gradient of circulation,

$$
\Gamma_{z}=\frac{1}{w}\left[\frac{1}{R e}\left(\Gamma_{r r}-\frac{\Gamma_{r}}{r}\right)-u \Gamma_{r}\right]
$$

Equations (8) and (A 4) are now used in (A 3) to arrive at a second-order, ordinary differential equation for $u$ in the independent variable $r$ :

$$
u_{r r}+\frac{u_{r}}{r}+u\left[\frac{1}{w}\left(\eta_{r}-\frac{\eta}{r}\right)+\frac{2 \Gamma \Gamma_{r}}{w^{2} r^{3}}-\frac{1}{r^{2}}\right]=\frac{1}{w R e}\left[\left(\eta_{r r}+\frac{\eta_{r}}{r}-\frac{\eta}{r^{2}}\right)+\frac{2 \Gamma}{w r^{3}}\left(\Gamma_{r r}-\frac{\Gamma_{r}}{r}\right)\right] .
$$

Equation (A 5) can be solved as a two-point boundary-value problem for $u(r)$ at a particular axial station for which $\psi(r)$ and $\Gamma(r)$ are known. $(w(r)$ and $\eta(r)$ are then also 
known through the definition of streamfunction, $(4 b)$, and the streamfunction equation, (6), respectively.)

The calculation of $u$ from (A 5) allows the determination of the axial gradients of $\psi$ and $\Gamma$ at particular stations. This calculation can be carried out in the transformed, computational domain by modifying (A 5 ) in an appropriate manner:

$$
u_{\zeta \zeta}+\frac{u_{\zeta}}{\zeta}+u\left[\left(\frac{1}{m w}\left(\eta_{\zeta}-\frac{\eta}{\zeta}\right)+\frac{2 m^{2} \Gamma \Gamma_{\zeta}}{w^{2} \zeta^{3}}-\frac{1}{\zeta^{2}}\right)\right]=\frac{1}{w R e}\left[\left(\eta_{\zeta \zeta}+\frac{\eta_{\zeta}}{\zeta}-\frac{\eta}{\zeta^{2}}\right)+\frac{2 m^{3} \Gamma}{w \zeta^{3}}\left(\Gamma_{\zeta \zeta}-\frac{\Gamma_{\zeta}}{\zeta}\right)\right] .
$$

Axial derivatives of $\eta$ and $\Gamma$ may be computed with

$$
\begin{gathered}
\psi_{\xi}=-r u-\dot{m} r \psi_{\zeta}=-r\left(u+\dot{m} \psi_{\zeta}\right), \\
\Gamma_{\xi}=\frac{1}{w}\left[\frac{m^{2}}{R e}\left(\Gamma_{\zeta \zeta}-\frac{\Gamma_{\zeta}}{\zeta}\right)-m u \Gamma_{\zeta}\right]-\dot{m} r \Gamma_{\zeta} .
\end{gathered}
$$

Based on (A 7) and (A 8), the following procedure is used to integrate the QC equations (the $j$ subscript is dropped for convenience):

(i) On a rectangular mesh in the $(\xi, \zeta)$-plane, the discrete quantities $\psi_{i}=\psi\left(\zeta_{i}\right)$ and $\Gamma_{i}=\Gamma\left(\zeta_{i}\right)$ are assumed known at a particular axial station. $w_{i}$ and $\eta_{i}$ are computed using second-order accurate, central-difference approximations to $(4 b)$ and $(6)$.

(ii) Solve (A 6) as a two-point, boundary-value problem for $u$,

$$
u_{\zeta \zeta}+\frac{1}{\zeta} u_{\zeta}+u f=g
$$

where $f$ and $g$ are obtained using central-difference approximations to derivatives of $\eta$ and $\Gamma$. Equation (A 9) is cast into discrete form using central-difference expressions and supplemented with the axial and radial boundary conditions

$$
\begin{aligned}
& u_{i}=0 \quad(\zeta=0), \\
& u_{i}=-\dot{m} \psi_{\zeta} \quad\left(\zeta=R_{0}\right) .
\end{aligned}
$$

The latter condition is approximated with a second-order accurate, onesided approximation.

(iii) $\psi_{\xi}$ and $\Gamma_{\xi}$ are computed at mesh points using (A 7) and (A 8).

(iv) $\psi$ and $\Gamma$ are computed at the next axial station with first-order accurate, Euler approximations:

$$
\begin{aligned}
& \psi_{i+1}=\psi_{i}+\Delta_{\xi} \psi_{\xi}+O\left[\Delta_{\xi}\right], \\
& \Gamma_{i+1}=\Gamma_{i}+\Delta_{\xi} \Gamma_{\xi}+O\left[\Delta_{\xi}\right] .
\end{aligned}
$$

\section{REFERENCES}

Anderson, D. A., Tannehtll, J. C. \& Pletcher, R. H. 1984 Computational Fluid Mechanics and Heat Transfer. Hemisphere.

Benjamin, T. B. 1962 Theory of the vortex breakdown phenomenon. J. Fluid Mech. 14, 593-629.

Benjamin, T. B. 1967 Some developments in the theory of vortex breakdown. J. Fluid Mech. 28, 65-84.

Beran, P. S. 1987 Numerical simulations of trailing vortex bursting. AIAA paper 87-1313.

BERAN, P. S. 1989 An investigation of the bursting of trailing vortices using numerical simulation. $\mathrm{PhD}$ thesis, California Institute of Technology. 
Braga, S. L. \& Hawthorne, W. R. 1950 Some exact solutions of the flow through annular cascade actuator discs. J. Aero. Sci. 17, 243-249.

Brown, G. I. \& Lopez, J. M. 1988 Axisymmetric vortex breakdown Part II: Physical mechanisms. Aero. Res. Lab. Aero. Rep. 174.

Escudier, M. P. 1984 Observations of the flow produced in a cylindrical container by a rotating endwall. Exps Fluids 2, 189-196.

FaLer, J. H. \& Leibovich, S. 1977 Disrupted states of vortex flow and vortex breakdown. Phys. Fluids 20, 1385-1400.

Faler, J. H. \& Leibovich, S. 1978 An experimental map of the internal structure of a vortex breakdown. J. Fluid Mech. 86, 313-335.

Grabowski, W. J. 1974 Solution of the Navier-Stokes equations for vortex breakdown. Berkeley Rep. FM-74-6.

Hafez, M., Ahmad, J., Kuruvila, G. \& Salas, M. D. 1987 Vortex breakdown simulation. AIAA paper 87-1343.

Hafez, M., Kuruvila, G. \& Salas, M. D. 1986 Numerical study of vortex breakdown. AIAA paper 86-0558.

HALL, J. L. 1985 An introduction to vortex breakdown and vortex core bursting. Natl Res. Council no. 24336.

Hall, M. G. 1967 A new approach to vortex breakdown. Proc. Heat Trans. Fluid Mech. Inst. pp. $319-340$.

Hall, M. G. 1972 Vortex breakdown. Ann. Rev. Fluid Mech. 4, 195-218.

Kellen, H. B. 1977 Numerical solution of bifurcation and nonlinear Eigenvalue problems. Applications of Bifurcation Theory, p. 359. Academic.

Kopecky, R. M. \& ToRrance, K. E. 1973 Initiation and structure of axisymmetric eddies in a rotating stream. Comput. Fluids, 1, 289.

Krause, E., Shi, X. G. \& Haktwich, P. M. 1983 Computation of leading-edge vortices. AIAA paper 83-1907.

Lambourne, N. C. \& Bryer, D. W. 1961 The bursting of leading-edge vortices-some observations and discussions of the phenomenon. Aero Res. Council RM-3282.

Leibovich, S. 1978 The structure of vortex breakdown. Ann. Rev. Fluid Mech. 10, 221-246.

Leibovich, S. 1984 Vortex stability and breakdown: survey and extension. AIAA J. 22, $1192-1206$.

LeIbovich, S. 1989 Vortex breakdown: a coherent transition trigger in concentrated vortices. Turbulence 89: Organized Structures and Turbulence in Fluid Mechanics. Kluwer.

LeIbovich, S. \& KRIBUS, A. 1990 Large-amplitude wavetrains and solitary waves in vortices. J. Fluid Mech. 216, 459-504.

Lopez, J. M. 1988 Axisymmetric vortex breakdown Part I : Confined swirling flow. Aero. Res. Lab. Aero. Rep. 173.

Lugt, H. J. \& Aвboud, M. 1987 Axisymmetric vortex breakdown with and without temperature effects in a container with a rotating lid. J. Fluid Mech. 179, 179-200.

Menne, S. 1988 Vortex breakdown in an axisymmetric flow. AIAA paper 88-0506.

Salas, M. D. \& Kuruvila, G. 1989 Vortex breakdown simulation: circumspect study of the steady, laminar, axisymmetric model. Comput. Fluids, 17, 247-262.

SARPKAYA, T. 1974 Effect of the adverse pressure gradient on vortex breakdown. AIAAJ. 12, 602 .

TÁaSAN, S. 1986 Multigrid method for a vortex breakdown simulation. NASA Contractor Rep. 178106.

Trigub, V. N. 1985 The problem of breakdown of a line vortex. Prikl. Math. Mech, 49, 166-171. 